\title{
Correlations between sunspots and their moat flows ${ }^{\star}$
}

\author{
J. Löhner-Böttcher and R. Schlichenmaier
}

\author{
Kiepenheuer-Institut für Sonnenphysik, Schöneckstr. 6, 79104 Freiburg, Germany \\ e-mail: [jlb;schliche]@kis.uni-freiburg.de
}

Received 11 October 2012 / Accepted 19 December 2012

\begin{abstract}
Context. The presence of the moat flow around sunspots is intimately linked to the mere existence of sunspots.

Aims. We characterize the moat flow (MF) and Evershed flow (EF) in sunspots to enhance our knowledge of sunspot structures and photospheric flow properties.

Methods. We calibrated HMI synoptic Doppler maps and used them to analyze $3 \mathrm{~h}$ time averages of 31 circular, stable, and fully developed sunspots at heliocentric angles of some $50^{\circ}$. Assuming axially symmetrical flow fields, we infer the azimuthally averaged horizontal velocity component of the MF and EF from 51 velocity maps. We studied the MF properties (velocity and extension) and elaborate on how these components depend on sunspot parameters (sunspot size and EF velocity). To explore the weekly and monthly evolution of MFs, we compare spots rotating from the eastern to western limbs and spots that reappear on the eastern limb.

Results. Our calibration procedure of HMI Doppler maps yields reliable and consistent results. In $3 \mathrm{~h}$ averages, we find the MF decreases on average from some $1000 \pm 200 \mathrm{~m} / \mathrm{s}$ just outside the spot boundary to $500 \mathrm{~m} / \mathrm{s}$ after an additional $4 \mathrm{Mm}$. The average MF extension lies at $9.2 \pm 5 \mathrm{Mm}$, where the velocity drops below some $180 \mathrm{~m} / \mathrm{s}$. Neither the MF velocity nor its extension depend significantly on the sunspot size or EF velocity. But, the EF velocity does show a tendency to be enhanced with sunspot size. On a time scale of a week and a month, we find decreasing MF extensions and a tendency for the MF velocity to increase for strongly decaying sunspots, whereas the changing EF velocity has no impact on the MF.

Conclusions. On $3 \mathrm{~h}$ averages, the EF velocity scales with the size of sunspots, while the MF properties show no significant correlation with the EF or with the sunspot size. This we interpret as a hint that the physical origins of EF and MF are distinct.
\end{abstract}

Key words. sunspots - Sun: activity - Sun: photosphere - Sun: rotation - convection - techniques: radial velocities

\section{Introduction}

As complex magnetic formations, sunspots strongly affects the photospheric dynamics of the granular convective pattern of the plasma in which they are embedded. They suppress the upward propagation of heat in the magnetic core, seen as the cooler and therefore darker umbra, and they harbor the well-known penumbral Evershed flow observed as a radially outwards-directed, horizontal flow in the photospheric layers. In photospheric observations this leads to a blueshift and blueward asymmetry of the spectral line for penumbral filaments facing the center of the solar disk and a redshift and redward asymmetry for filaments facing the solar limb (e.g., Evershed 1909; Maltby 1964; Schlichenmaier et al. 2004). In general, the line shift, as well as the asymmetry, increases from the inner to the outer penumbra. In photospheric layers the Evershed flow is close to horizontal with maximum inclinations of $5^{\circ}$ to $10^{\circ}$, exhibiting downward flows in the outer penumbra (e.g., Michard 1951; Balthasar et al. 1996; Schlichenmaier \& Schmidt 2000; Franz $\&$ Schlichenmaier 2009). Average horizontal velocities are in the range of 3-4 $\mathrm{km} \mathrm{s}^{-1}$ (Shine et al. 1994), but can exceed $6 \mathrm{~km} \mathrm{~s}^{-1}$ or more on small scales (e.g., Rouppe van der Voort 2002; Bellot Rubio et al. 2003a). The Evershed flow seems to stop abruptly at the white light boundary of the spot (Wiehr \& Degenhardt 1992; Schlichenmaier \& Schmidt 1999), which is in line with downward flows in the outer penumbra. Only a small fraction of the flow continues to flow into the magnetic canopy that surrounds the spot (Rezaei et al. 2006).

\footnotetext{
* Appendix $\mathrm{A}$ is available in electronic form at http://www. aanda.org
}

Whereas the Evershed flow (EF) is magnetized (e.g., Schlichenmaier \& Collados 2002), the annular region around fully-fledged sunspots, called the moat cell, is largely nonmagnetic. It harbors the moat flow (MF), a radially oriented outflow of plasma adjacent to the outer penumbral boundary. Embedded in these motions, small, moving magnetic features (MMFs) migrate away from the sunspot (Sheeley 1969, 1972; Vrabec 1971; Harvey \& Harvey 1973). The existence of both the MF and the EF depend on the presence of a penumbra (Vrabec 1974). Consequently, the moat flow can only be observed for sunspot sides with a well-developed penumbra and lacks pores (Sobotka et al. 1999).

The moat flow develops immediately after the formation of a penumbra (Pardon et al. 1979; Schlichenmaier et al. 2010) and was observed even after the decay of the penumbra (Verma et al. 2012). The MF is present as a stationary flow through the spot's lifetime, which varies between several weeks to several months (see, e.g., Solanki 2003): Sobotka \& Roudier (2007) do not find any significant variations in the MF within half a day. During the initiation of the MF, the magnetic components in the vicinity of the sunspot are pushed to the periphery and leave the moat cell largely nonmagnetic with single MMFs. The extension of the moat field reaches between $10 \mathrm{Mm}$ to $20 \mathrm{Mm}$ from the penumbral boundary for small sunspots and can be roughly twice the spot radius for larger spots (Brickhouse \& Labonte 1988), but Sobotka \& Roudier (2007) find no such correspondence.

The moat flow velocity ranges between $0.5 \mathrm{~km} \mathrm{~s}^{-1}$ to $1 \mathrm{~km} \mathrm{~s}^{-1}$ and can be seen by tracking the bright granular features (e.g., Rimmele 1997; Vargas Domínguez et al. 2007; Balthasar \& Muglach 2010) or Doppler shift measurements 
(Balthasar et al. 1996). That the MF consists of migrating granules, indicates that the MF is as nonmagnetic as granules, i.e., largely nonmagnetic.

Also helioseismic measurements have revealed the existence of the MF as an outflow extending up to $30 \mathrm{Mm}$ with a maximum velocity of $1 \mathrm{~km} \mathrm{~s}^{-1}$ just next to the penumbral boundary (Gizon et al. 2000) in the first $2 \mathrm{Mm}$ of the solar surface. According to recent studies (Sun et al. 1997; Gizon et al. 2009; Featherstone et al. 2011), the MF is also detectable in deeper layers, but has slower speeds compared to surface measurements.

Cabrera Solana et al. (2006) suggest that a link exists between EF and MMFs. Magnetic velocity packages, called the Evershed clouds inside the penumbra (Shine et al. 1994; Rimmele 1994; Cabrera Solana et al. 2007) propagate outwards to the extension of penumbral filaments and the moat region where they are embedded as MMFs. MMFs can travel from the penumbra into the vicinity of the sunspot (Sainz Dalda \& Martínez Pillet 2005; Sainz Dalda \& Bellot Rubio 2008a,b). Also Schlichenmaier (2002) proposes a scenario that is consistent with observed MMFs. In this way, a magneto-convective overshoot instability in an Evershed flux tube leads to the migrating feature in the MF region. There is still discordance about a link between the EF and MF. Vargas Domínguez et al. (2007) observed irregular sunspots and found that the moat flow is only present in radial extensions of penumbral filaments, but not perpendicular to them. This indicates that the MF is an extension of the EF. However, this seems to be impossible since the EF is magnetized, while the MF is intrinsically unmagnetic, as mentioned above.

In this paper, we analyze the EF and MF and elaborate on a possible link between the two. To that end, we utilize Doppler shift measurements of HMI. In Sect. 2 we perform a thorough calibration of HMI Doppler maps. In Sect. 3 we describe the criteria for data selection and the method we used to analyze the flow properties. In Sect. 4 we present our results for the flow properties and elaborate on correlations between spot and MF properties and on how they change during the evolution of a spot. The statistics are based on 51 maps constructed from 31 different spots. The data sets we used and the radial dependencies of the flow velocity of all spots, which form the basis of our analysis, are given in the appendix. In Sect. 5, we discuss the present understanding of MFs in the context of our results and summarize our results and conclusions.

\section{Calibration of HMI Doppler maps}

Data: our work is based on synoptic $720 \mathrm{~s}$ intensity maps and Doppler maps ${ }^{1}$ in the Fe I spectral line at $6173.3 \AA$ from the JSOC webpage recorded by the Helioseismic Magnetic Imager (HMI) of the Solar Dynamics Observatory (SDO) up to maximum values of $\pm 6.5 \mathrm{~km} \mathrm{~s}^{-1}$ and a spatial resolution of $\approx 0.5^{\prime \prime} / \mathrm{px}$.

Calibration by subtracting systematic components: to yield undisturbed flow velocities relative to the solar surface, we need to define a rest frame $(v=0)$. To this end, we construct a time averaged velocity map, $\langle v(x, y)\rangle_{t}$, in which the effects of granulation, oscillations, and supergranulation are removed. This average is composed of three systematic, large-scale components:

$\langle v(x, y)\rangle_{t}=v_{\mathrm{clv}}(r)+v_{\text {rot }}\left(d, l, B_{0}\right)+v_{\text {res }}(x, y)$.

\footnotetext{
1 A velocity map of the Sun according to spectral line shifts due to the Doppler effect.
}

The first term is the center-to-limb variation in the convective blueshift, $v_{\text {clv }}$. It is radially symmetrical and is several hundred $\mathrm{m} / \mathrm{s}$, depending on the distance, $r$, (or heliocentric angle $\theta$ ) to disk center. The second term is the differential rotation, $v_{\text {rot }}$. It is axially symmetrical with velocities up to $\pm 2000 \mathrm{~m} / \mathrm{s}$ with respect to the meridian. The values depend on the distance $d$ from the meridian, the latitude $l$ and the inclination $B_{0}$ of the rotation axis, which varies annually between $\pm 7.27^{\circ}$. The third term is a nonsymmetrical residual, $v_{\text {res }}$, with velocities up to $\pm 150 \mathrm{~m} / \mathrm{s}$, which contains instrumental effects and other systematic flow fields of smaller magnitude. The large-scale meridional flow with velocities of less than $20 \mathrm{~m} / \mathrm{s}$ can be neglected for our sunspot studies, and if existing, it is included in these three components. In the next four sections we determine the four terms of Eq. (1).

\subsection{Construction of a time-averaged velocity map: $\langle v(x, y)\rangle_{t}$}

In velocity maps, supergranules produce a redshift on the limb side and a blueshift on the opposite side when they are off disk center. Because a supergranule migrates across the disk its average velocity signal vanishes when it is next to the equator, since blueshifts are eliminated by the following redshifts. However, close to the poles, the blueshifts and redshifts are separated in latitude such that a single supergranule leaves noticeable traces in the Doppler map. To diminish these traces a long time series is necessary. In our case we have averaged $720 \mathrm{~s}$ Doppler maps of 61 entire days (a total of 7320 maps) between June and December 2010, shown in the left hand panel of Fig. 1.

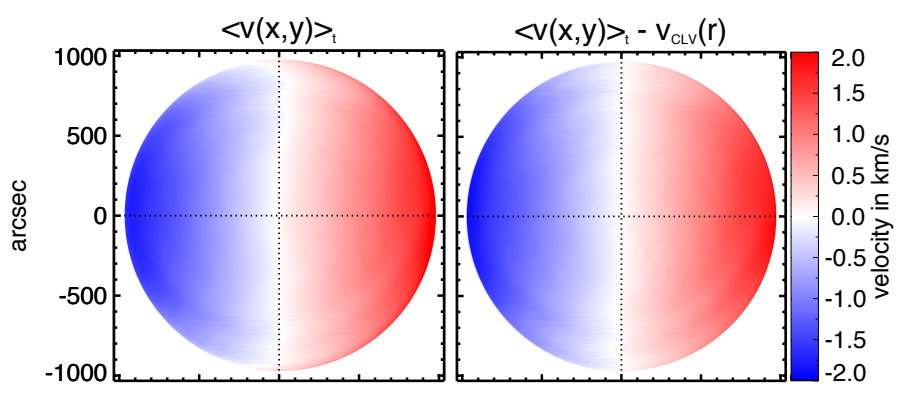

Fig. 1. Left panel: averaged velocity map, $\langle v(x, y)\rangle_{t=61 \text { days }}$, with maximum blueshifts (eastern limb) and redshifts (western limb) with some $2 \mathrm{~km} \mathrm{~s}^{-1}$. The axes are given in arcsec. Right panel: reduced velocity map, $\langle v(x, y)\rangle_{t}-v_{\text {clv }}$.

In order not to spoil the time average by active regions, we have masked regions according to the darkening in the continuum intensity and replaced the content of the mask in the Doppler map by the velocities on the opposite side of the heliocentric equator. Thanks to the solar activity minimum, this method was appropriate. For consistency, we normalized the solar radius of all Doppler maps and eliminated the respective observer motion ${ }^{2}$ relative to the Sun. Since we favored time periods with inclination $B_{0} \approx 0^{\circ}$, the obtained velocity map $\langle v(x, y)\rangle_{t=61 \text { days }}$ should serve as a good basis for modeling the systematic components named in Eq. (1). In $\langle v(x, y)\rangle_{t}$ the velocity at disk center is about $50 \mathrm{~m} / \mathrm{s}$.

\footnotetext{
2 Keywords for observer motion in the FITS file header: $O B S \_V R$ (radial), $O B S_{-} V W$ (westward), and $O B S_{-} V N$ (northward).
} 


\subsection{Radially symmetrical velocity component: $v_{\mathrm{clv}}(r)$}

As seen in the left hand panel of Fig. 1, the LOS velocity of the solar rotation, which should be zero for the meridian and axially symmetrical with respect to the centerlines, is deformed. This deformation along the meridian contains the center-to-limb variation $(\mathrm{CLV})$ of the convective blueshift and the meridional flow. To determine the sum of both effects a priori (without knowing the differential rotation), we decided to use the average, $\langle v(0 \pm \mathrm{d} x, y)\rangle_{t ; \mathrm{d} x=10 \mathrm{px} ; \pm y}$, of a 20-pixel wide stripe along the meridian of $\langle v(x, y)\rangle_{t}$ with respect to the distance, $r$, to the disk center as shown in Fig. 2a. Assuming radial symmetry, the curves of the northern and southern hemispheres are averaged. The resulting $\langle v(0, y)\rangle_{t}$ is fitted by a 8 th-order polynomial, $v_{\mathrm{clv}}(y)$. The curve runs from $+50 \mathrm{~m} / \mathrm{s}$ at the disk center to $-60 \mathrm{~m} / \mathrm{s}$ at some $700^{\prime \prime}$ and strong redshifts exceeding $300 \mathrm{~m} / \mathrm{s}$ at the solar limb. This CLV is in good accordance with recent studies of the CLV of the Fe I line (e.g., Balthasar 1985; Cruz Rodríguez et al. 2011). As the up- and downturns, which are caused by the supergranular migration at higher latitudes, are on the order of some $30 \mathrm{~m} / \mathrm{s}$, we neglect the impact of the meridional flow $^{3}$ on $v_{\mathrm{clv}}(y)$ and further calibrations. We use the polynomial $v_{\mathrm{clv}}(y)$ to create a radially symmetrical CLV model, $v_{\text {clv }}(r)$, as displayed in Fig. 2b.

Since the convective blueshift is the result of the bigger impact of hot upwards moving granules than the cool, downwards directed, intergranular fraction on a spatially averaged solar surface, the line shift depends on the observed spectral line and the line of sight. According to calculations based on the Liège atlas (Delbouille et al. 1990), the convective blueshift of the Fe $\mathrm{I}$ spectral line at $6173.3 \AA$ in the center is $v_{\mathrm{b}}=-305 \mathrm{~m} / \mathrm{s}$. For observational studies in Sect. 3, we have to offset $v_{\text {clv }}(r)$ by $v_{\text {off }} \approx-350 \mathrm{~m} / \mathrm{s}$ to obtain the convective blueshift, $v_{\mathrm{b}}(r)$, with its CLV.

After the calibration of $v_{\text {rot }}\left(d, l, B_{0}\right)$ and $v_{\text {res }}(x, y)$ in Sects. 2.3 and 2.4 we can check the accuracy of the data calibration by computing the CLV velocity map a posteriori:

$\tilde{v}_{\mathrm{clv}}(x, y)=\langle v(x, y)\rangle_{t}-v_{\text {rot }}\left(d, l, B_{0}\right)-v_{\text {res }}(x, y)$.

Then, we compare the CLV in the meridional and equatorial directions, i.e. $\left\langle\tilde{v}_{\mathrm{clv}}(0 \pm \mathrm{d} x, y)\right\rangle_{\mathrm{d} x=10 \mathrm{px} ; \pm y}$ and $\left\langle\tilde{v}_{\mathrm{clv}}(x, 0 \pm \mathrm{d} y)\right\rangle_{\mathrm{d} y=10 \mathrm{px} ; \pm x}$. The curves are displayed in Fig. 2c and closely resemble each other. The velocities were offset by $v_{\text {off }}$ to achieve the run from $v_{\mathrm{b}}(\cos \theta=1)=-305 \mathrm{~m} / \mathrm{s}$ to $v_{\mathrm{b}}(\cos \theta=0.8 \ldots 0.6) \approx-400 \mathrm{~m} / \mathrm{s}$ (preferred spot location) and lower velocities at the solar limb. While the equatorial direction exhibits minor fluctuations, the meridional direction displays stronger fluctuations owing to the residuals of supergranules. The difference between both curves shown in the upper part of Fig. 2c reveals an rms smaller than $10 \mathrm{~m} / \mathrm{s}$ in low latitudes. The dashed green curve in Fig. 2c results from synthetic profiles for Fe I $6173.3 \AA$ based on COBOLD simulations (Freytag et al. 2012; Beeck et al. 2012). The synthetic curve differs by less than $100 \mathrm{~m} / \mathrm{s}$, so is in good qualitative agreement with our measurements.

\subsection{Differential rotation: $v_{\text {rot }}\left(d, I, B_{0}\right)$}

Since the rotation velocity is differential on the solar surface and depends on the line of sight (LOS), we have to generate a specific, axially symmetrical solar rotation model, $v_{\text {rot }}\left(d, l, B_{0}\right)$, with

\footnotetext{
3 A large-scale axisymmetrical flow directing from the equator to the poles on the solar surface with velocities up to $20 \mathrm{~m} / \mathrm{s}$ at latitudes around $35^{\circ}$ (Hathaway \& Rightmire 2010; Komm et al. 2011).
}

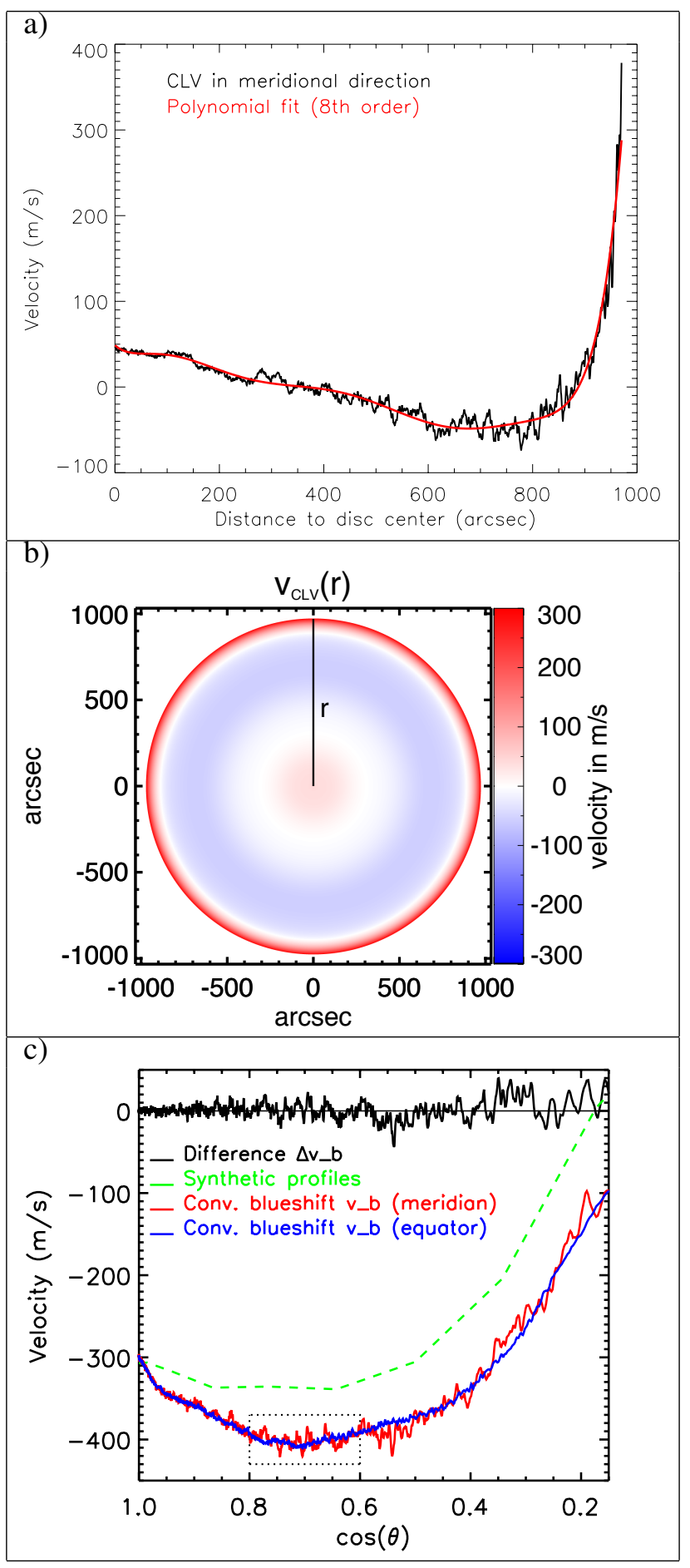

Fig. 2. a) Average $\operatorname{CLV},\langle v(0, y)\rangle_{t}$, along the meridional centerline with an 8 th-order polynomial fit, $v_{\text {clv }}(y)$ (red curve) in $\mathrm{m} / \mathrm{s}$ against the distance $y$ to the disk center in arcsec. b) Calibrated, radially symmetrical CLV model, $v_{\text {clv }}(r)$, with axis given in arcsec. c) Convective blueshift, $v_{\mathrm{b}}(r)$, in $\tilde{v}_{\text {clv }}$ along the meridian (red solid curve) and equator (blue solid curve) and their difference (black solid curve) displayed in $\mathrm{m} / \mathrm{s}$ against $\cos \theta$ with heliocentric angle, $\theta$. A synthesis of the spectral line from COBOLD simulations (green dashed curve) is added. The dotted black box refers to the preferred sunspot location.

respect to the distance, $d$, from the meridian, the latitude, $l$, and the inclination, $B_{0}$, of the rotation axis. 


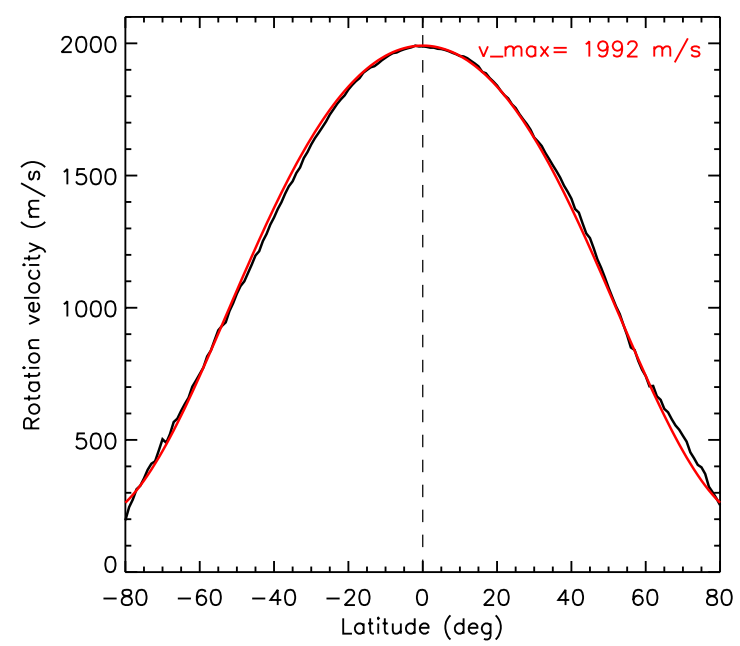

Fig. 3. Absolute rotation velocities, $v_{\text {rot }}\left(l, B_{0}=0\right.$ ), in $\mathrm{m} / \mathrm{s}$ (black curve) on the solar surface for latitudes up to $l= \pm 80^{\circ}$ fitted by $\Omega(l)$ (red curve) and an equatorial maximum of $\Omega(0)=1992 \mathrm{~m} / \mathrm{s}$.

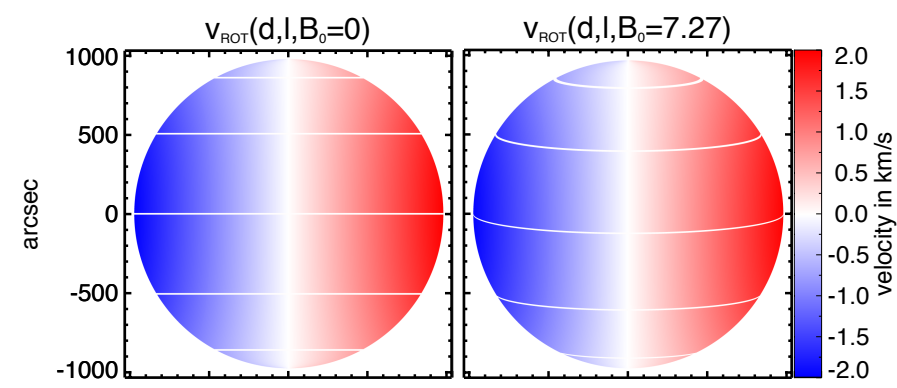

Fig. 4. Computed differential rotation maps, $v_{\text {rot }}\left(d, l, B_{0}=0^{\circ}\right.$ ) (left panel) and $v_{\text {rot }}\left(d, l, B_{0}=7.27^{\circ}\right)$ (right panel), with maximum velocities $v_{\text {rot }}\left(l=0, B_{0}=0^{\circ}\right)=1992 \mathrm{~m} / \mathrm{s}$ and $v_{\text {rot }}\left(l=0, B_{0}=7.27^{\circ}\right)=1976 \mathrm{~m} / \mathrm{s}$, spatially displayed in arcsec.

We start out with the time-averaged velocity map reduced by the radially symmetrical component, i.e. $\langle v(x, y)\rangle_{t}-v_{\mathrm{clv}}(r)$, displayed in the right hand panel of Fig. 1. We determine the absolute latitudinal velocity, $v_{\text {rot }}(l)$ for $B_{0}=0^{\circ}$, by performing linear regressions for the LOS velocities along latitudinal cuts, $v\left(\mathrm{~d} / R_{l}\right)$, with $R_{l}$ being the distance of the solar limb from the meridian: $v_{\text {rot }}(d, l)=v_{\text {rot }}(l) \cdot\left(d / R_{l}\right)$. Figure 3 shows the latitudinal dependence, $v_{\text {rot }}(l)$.

To compare our rotation model with literature values, we follow Stix (2002) and approximate $v_{\text {rot }}(l)$ by

$\Omega(l)=\mathrm{A}+\mathrm{B} \sin ^{2} l+\mathrm{C} \sin ^{4} l$.

The approximation, $\Omega(l)$, is plotted in Fig. 3. We obtained a differential decrease to higher latitudes. The equatorial maximum, $\mathrm{A}=14.10 \pm 0.03^{\circ} / \mathrm{day}$, corresponds to $\Omega(0)=1992 \mathrm{~m} / \mathrm{s}$. The coefficients $B=-9.0^{\circ} /$ day and $C=-2.5^{\circ} /$ day have standard deviations on the order of $0.1 \%$ day. Since the result depends on the method of measurement (see: Stix 2002), we compared our rotational velocity with the Doppler shift measurements of Snodgrass (1984) who obtained an equatorial rate of $A=14.05 \pm 0.01^{\circ} /$ day or $\Omega(0)=1975 \mathrm{~m} / \mathrm{s}$. This is in good accordance with our findings. However, compared to Snodgras coefficients, $B=-1.49^{\circ} /$ day and $C=-2.61^{\circ} /$ day with deviations on the order of some $0.1^{\circ} /$ day, we obtained a stronger decrease in the rotational velocity to higher latitudes.

The differential rotation model, $v_{\text {rot }}\left(d, l, B_{0}\right)$, displayed as an example in Fig. 4 for $B_{0}=0^{\circ}$ (left panel) and $B_{0}=7.27^{\circ}$ (right panel), generates the LOS velocities for every position on the solar disk according to the annually varying $B_{0}= \pm 7.27^{\circ}$.

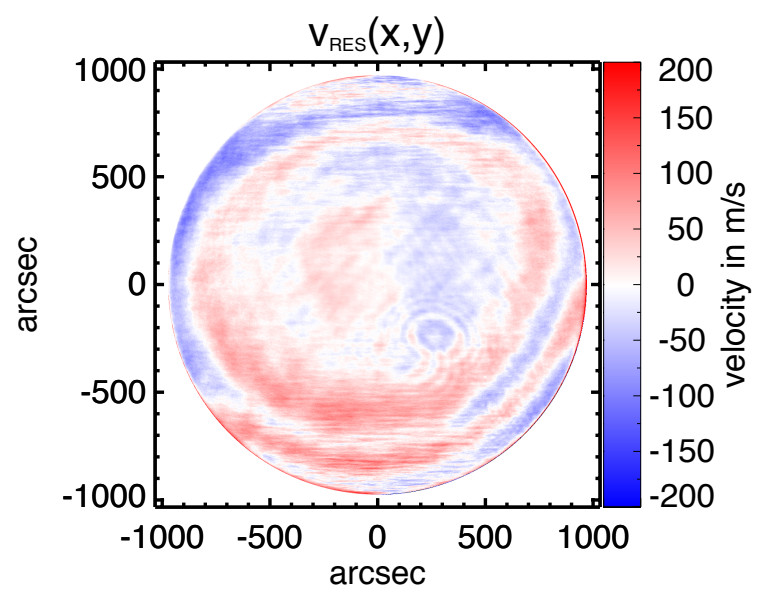

Fig. 5. Residual velocity map, $v_{\text {res }}(x, y)$, with axis given in arcsec.

\subsection{Residual velocity map: $v_{\text {res }}(x, y)$}

Augural annular patterns (see Fig. 1) indicate a systematical, nonsymmetrical residual, $v_{\text {res }}(x, y)$, that contains instrumental effects and other systematic flow fields of smaller magnitude. Subtracting $v_{\text {clv }}$ and $v_{\text {rot }}$ from the 61 day average, $\langle v\rangle_{t}$, we obtain the residual nonsymmetrical velocity map:

$v_{\text {res }}(x, y)=\langle v(x, y)\rangle_{t}-v_{\text {clv }}(r)-v_{\text {rot }}\left(d, l, B_{0}=0^{\circ}\right)$,

which is displayed in Fig. 5. Our result agrees well with the HMI calibration studies of Howe et al. (2011) and Centeno et al. (2011). Several large circular patterns and smaller interference rings were checked as spatially fixed with velocities varying between $\pm 150 \mathrm{~m} / \mathrm{s}$. The residual map is subtracted a priori for all Doppler maps in Sect. 3. Changes in time are expected to have small amplitude so will not affect our analysis of horizontal flow velocities.

\section{Data selection and analysis}

Overview: in this section, we analyze the sunspot moat flow and Evershed flow in the solar photosphere by the calibrated 720 s Doppler maps recorded by HMI. We have selected circular sunspots and constructed $3 \mathrm{~h}$ time averages (out of 15 such Doppler maps) of the field of view (FOV). By doing this, we reduce the amplitudes of granulation and oscillation (see Sect. 3.1). We analyze the flow field in the penumbra and in the surrounding moat cell.

\subsection{Data selection and preprocessing:}

The moat flow is coupled to the presence of a penumbra and follows the direction of the penumbral filaments. Thus, we searched for sunspots with a fully-fledged, circular penumbra and found 31 applicable, single sunspots in a slight decay ${ }^{4}$ in the time between June 2010 and January 2012. The selected sunspots vary in size between $9 \mathrm{Mm}$ and $22 \mathrm{Mm}$ and are listed in Table A.1 of the appendix. A contour of the outer penumbra for each spot is determined by means of an intensity threshold for a spatially averaged image. The center of a sunspot is determined as the center-of-gravity of all points inside its penumbral contour.

To analyze predominantly horizontal flows with LOS Doppler maps, we selected the observing position of the sunspot center at heliocentric angles of some $\theta \approx 50^{\circ}$. There, the LOS

4 Zürich Classification: Group H. 

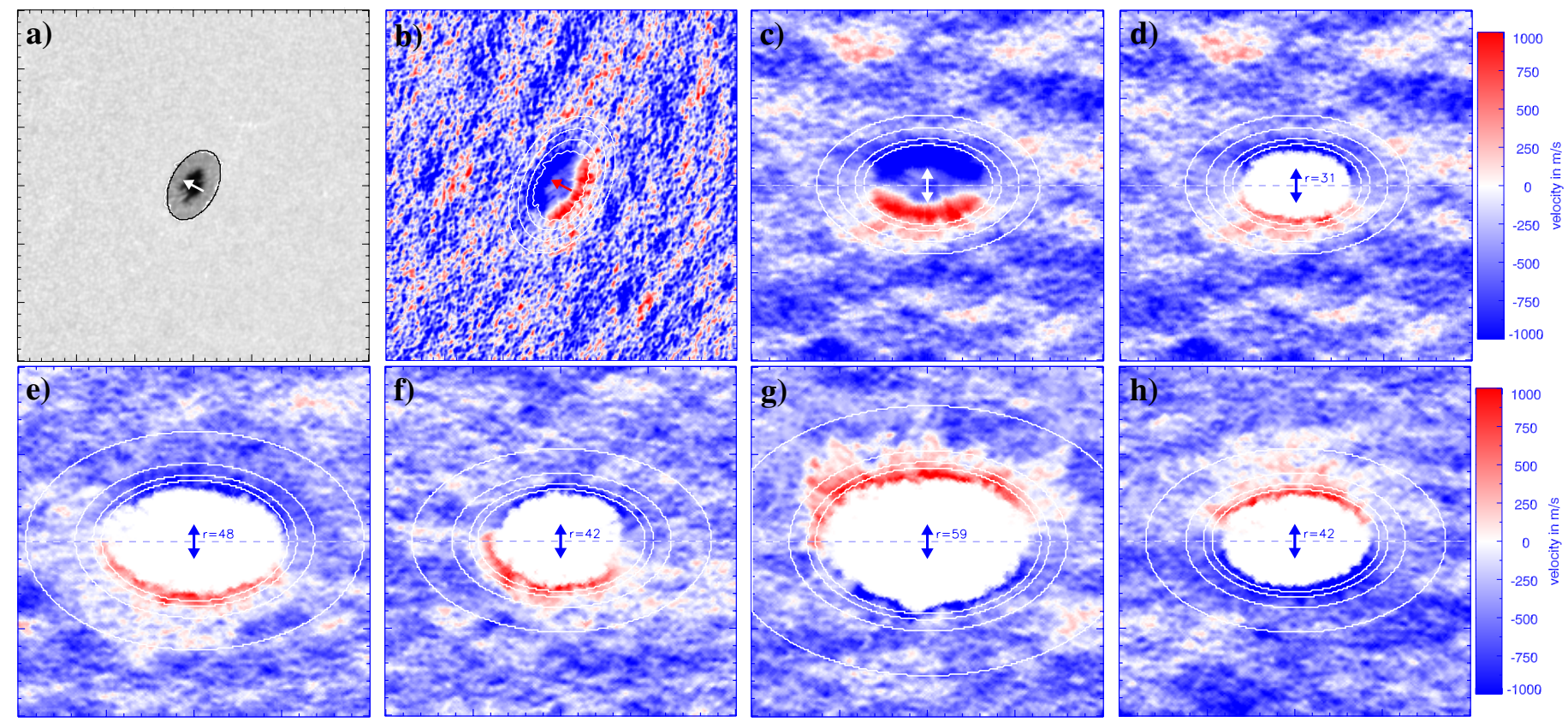

Fig. 6. a) $720 \mathrm{~s}$ intensity map and b) corresponding $720 \mathrm{~s}$ velocity map, $\tilde{v}_{\mathrm{dop}}$, of AR 11084 located at $19^{\circ} \mathrm{S}, 48^{\circ} \mathrm{W}$, respectively $\theta=51^{\circ}$, recorded 2010 July 6 at 01:36 UT (see Table A.1: No. 1b). An arrow is pointing to the disk center. The penumbral contour (white line), the theoretical, circular spot shape (black ellipse), and foreshortened circles with rising radii are displayed as well. The FOV size is $301 \mathrm{px}\left(\approx 150^{\prime \prime}\right)$. c) Aligned $3 \mathrm{~h}$ average map, $v_{3 \mathrm{~h}}$, of AR11084. d-h) Masked versions of spots No. 1b, 4a, 6a, 7a, 9a (Table A.1) at a FOV size of $201 \mathrm{px}\left(\approx 100^{\prime \prime}\right)$ and white ellipses with $R_{\mathrm{S}}+\left.x\right|_{x=5,10,20, R_{\mathrm{S}}}$. The velocities are displayed up to $\pm 1000 \mathrm{~m} / \mathrm{s}$.

component of the horizontal flow is significant, while the calibration uncertainties that increase towards the solar limb are still small.

Each sunspot FOV from the size of $301 \times 301$ px is tracked by its averaged center, $\left(x_{\mathrm{c}}, y_{\mathrm{c}}\right)$, for 15 successive $720 \mathrm{~s}$ intensity maps (Fig. 6a), while the respective Doppler maps, $v_{\text {dop }}(x, y)$, are calibrated by

$\tilde{v}_{\text {dop }}(x, y)=v_{\text {dop }}(x, y)-v_{\text {rot }}\left(d, l, B_{0}\right)-v_{\text {res }}(x, y)+v_{\text {off }}(x, y)$

to obtain velocity maps, $\tilde{v}_{\text {dop }}(x, y)$, (Fig. $\left.6 \mathrm{~b}\right)$ free of all systematical velocity components (described in Sect. 2), but the surface convection. $v_{\text {off }}$ describes the offset of the convective blueshift and its CLV (Fig. 2c) to values around $-400 \mathrm{~m} / \mathrm{s}$ at the chosen observing position.

The sunspot and flow properties are stable within three hours. To diminish 5-min oscillations and granulation, we averaged the tracked and aligned sunspot FOVs $(201 \times 201 \mathrm{px})$ of all the 15 successive velocity maps and refer to this $3 \mathrm{~h}$ average as $v_{3 \mathrm{~h}}$ (Figs. $6 \mathrm{c}-\mathrm{h}$ ). Likewise, the received LOS velocities are adapted for an average heliocentric angle, $\theta$. For better illustrating the MF, the $3 \mathrm{~h}$ average is shown here with a masked umbra and penumbra.

We studied the weekly evolution of the observed components by tracking 20 sunspots near the eastern limb to a second proper position $\left(\theta \approx 50^{\circ}\right)$ after some 6 to 8 days and compared the results of both $v_{3 \mathrm{~h}}$ analysis. As this was not possible for 11 of 31 spots, we achieve a sample of 51 velocity maps. Tracking three longlasting sunspots across the far side of the Sun, they reappear at the eastern front side (Table A.1: No. $4 \rightarrow 6,7 \rightarrow 9,11 \rightarrow 13$; Figs. 6e-h) which allows us to study the monthly evolution.

\subsection{Method of flow analysis}

We analyze flow fields of fully calibrated velocity maps, $v_{3 \mathrm{~h}}$, by applying a method that determines the azimuthally averaged flow properties (Schlichenmaier \& Schmidt 2000). In this way, we assume axially symmetrical flow fields for circular sunspots with a fully developed penumbra. The foreshortening effects of a circular sunspot at a certain heliocentric angle, $\theta$, cause the elliptical shape as shown for AR11084 (Figs. 6a-d). To observe the spatial velocity dependency of the flow fields, we use an automatic procedure that generates ellipses as foreshortened circles (with radii, $r$, in px) based on the heliocentric angle and rotated according to their position on the disk. The LOS velocities, $v^{\operatorname{LOS}}(r, \phi)$, along the ellipses are read out according to their circular angle, $\phi$, as shown in Fig. 7 for distances $r=R_{\mathrm{S}}+5$ (upper panel) and $r=R_{\mathrm{S}}+10$ (lower panel) from the spot center. The sunspot radius is automatically determined by the minimal deviation of the ellipse to the penumbral contour for every $720 \mathrm{~s}$ intensity map and averaged for the $3 \mathrm{~h}$ period. A manual fine adjustment was added for irregularities. The LOS velocities, $v^{\mathrm{LOS}}(r, \phi)$, were fitted by a sine function:

$v^{\mathrm{LOS}}(r, \phi)=v_{\|, 0}^{\mathrm{LOS}}(r) \cdot \sin \phi+v_{\perp}^{\mathrm{LOS}}(r)$

The amplitude, $v_{\|, 0}^{\mathrm{LOS}}$, is the LOS component of the horizontal flow velocity

$$
v_{\|, 0}(r)=v_{\|, 0}^{\mathrm{LOS}}(r) / \sin \theta
$$

at the heliocentric angle, $\theta$. The axial offset, $v_{\perp}^{\mathrm{LOS}}$, is the vertical flow component.

\subsection{Moat flow}

We analyze the moat flow in the vicinity of the sunspots for the fully calibrated velocity maps, $v_{3 \mathrm{~h}}$ (listed in Table A.1).

\subsubsection{Moat flow velocity}

The moat flow is an outwardly directed flow, which is predominantly horizontal. We present the flow field analysis for the 


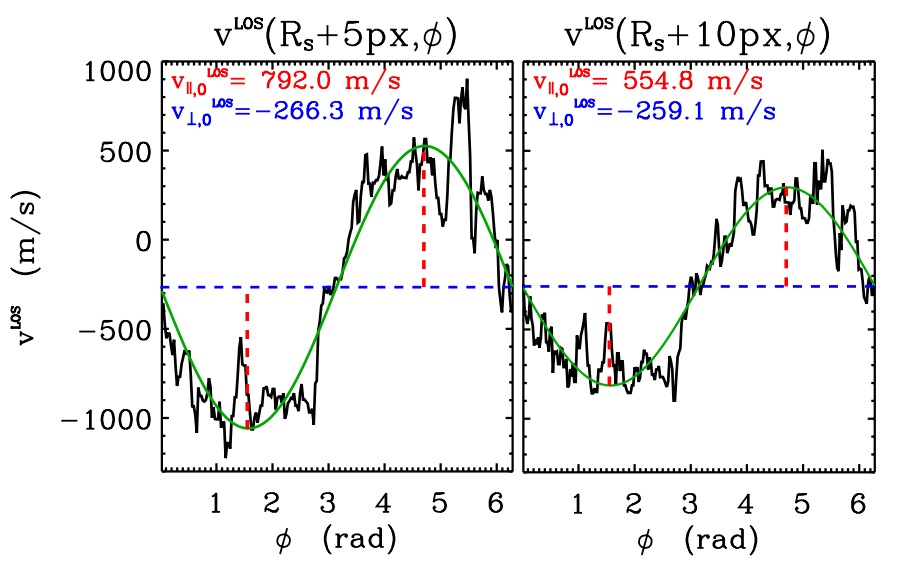

Fig. 7. Angular LOS velocities, $v^{\mathrm{LOS}}(r, \phi)$, in $\mathrm{m} / \mathrm{s}$ along the elliptical projection for $\theta=51^{\circ}$ of circles with radius $r=R_{\mathrm{S}}+5 \mathrm{px}$ (left panel) and $r=R_{\mathrm{S}}+10 \mathrm{px}$ (right panel) fitted by a sine curve (green, solid line) according to Eq. (5). The amplitude, $v_{\|, 0}^{\mathrm{LOS}}(r)$, and offset, $v_{\perp}^{\mathrm{LOS}}(r)$ are marked by red and blue dashed lines.

velocity map, $v_{3 h}$, of AR11084 (Figs. 6c, d; Table A.1: No. 1b). The sunspot in the FOV center with an average spot radius of $R_{\mathrm{S}}=31 \mathrm{px} \approx 11.2 \mathrm{Mm}$ at $\theta=51^{\circ}$ exhibits a distinct moat flow surrounding the sunspot with LOS velocities up to $-1000 \mathrm{~m} / \mathrm{s}$ for the blueshifted side facing the disk center and $+1000 \mathrm{~m} / \mathrm{s}$ for the redshifted limb side indicated by an arrow. The outer vicinity of the sunspot features supergranular flow cells.

Comparing the velocity components of the MF in a distance of $5 \mathrm{px}$ and $10 \mathrm{px}$ from the sunspot (see Fig. 7)

$v_{\|, 0}^{\mathrm{LOS}}\left(R_{\mathrm{S}}+5 \mathrm{px}\right)=-792 \pm 17 \mathrm{~m} / \mathrm{s}$

$v_{\|, 0}^{\mathrm{LOS}}\left(R_{\mathrm{S}}+10 \mathrm{px}\right)=-555 \pm 12 \mathrm{~m} / \mathrm{s}$

$v_{\perp}^{\mathrm{LOS}}\left(R_{\mathrm{S}}+5 \mathrm{px}\right)=-266 \pm 12 \mathrm{~m} / \mathrm{s}$

$v_{\perp}^{\mathrm{LOS}}\left(R_{\mathrm{S}}+10 \mathrm{px}\right)=-259 \pm 9 \mathrm{~m} / \mathrm{s}$,

the most prominent result is the decrease in $v_{\|, 0}^{\mathrm{LOS}}$ by more than $230 \mathrm{~m} / \mathrm{s}$ within these $2 \mathrm{Mm}$. The upper panel of Fig. 8 shows the continuous run of the horizontal flow velocities, $v_{\|, 0}(r)$, at $\theta=51^{\circ}$ (see Eq. (6)), from the spot center to a distance of $25 \mathrm{Mm}$. The MF has a strong monotone decrease with increasing distance from $1150 \mathrm{~m} / \mathrm{s}$ just outside the penumbra to $600 \mathrm{~m} / \mathrm{s}$ after additional $3 \mathrm{Mm}$ and $250 \mathrm{~m} / \mathrm{s}$ after $6 \mathrm{Mm}$.

The vertical flow component of MF and EF depends on the choice of the rest frame. Our rest frame is determined by the convective blueshift of nonmagnetic convection. Since the MF region is not completely free of magnetic fields, it may change the convective blueshift by an unknown amount. Therefore, no accurate analysis of the vertical MF component, $v_{\perp}^{\mathrm{LOS}}(r)$, can be given. Also, the inclination of the MF could not be reliably determined owing to the dependence on the vertical flow component (Schlichenmaier \& Schmidt 2000). However, on the assumption that no effect is caused by magnetic fields, we can subtract the convective blueshift $v_{\mathrm{b}}(r) \approx-400 \mathrm{~m} / \mathrm{s}$ from $v_{\perp}^{\mathrm{LOS}}(r)$ and obtain absolute vertical velocities of $\tilde{v}_{\perp}^{\mathrm{LOS}}(r)=0 \ldots 200 \mathrm{~m} / \mathrm{s}$. According to that, the slight redshift indicates a downwarddirected MF. In comparison with horizontal MF velocities up to $v_{\|, 0}^{\mathrm{LOS}}=1000 \mathrm{~m} / \mathrm{s}$, the vertical velocity is an order of magnitude less, and therefore it has a minor impact on the absolute flow velocity.
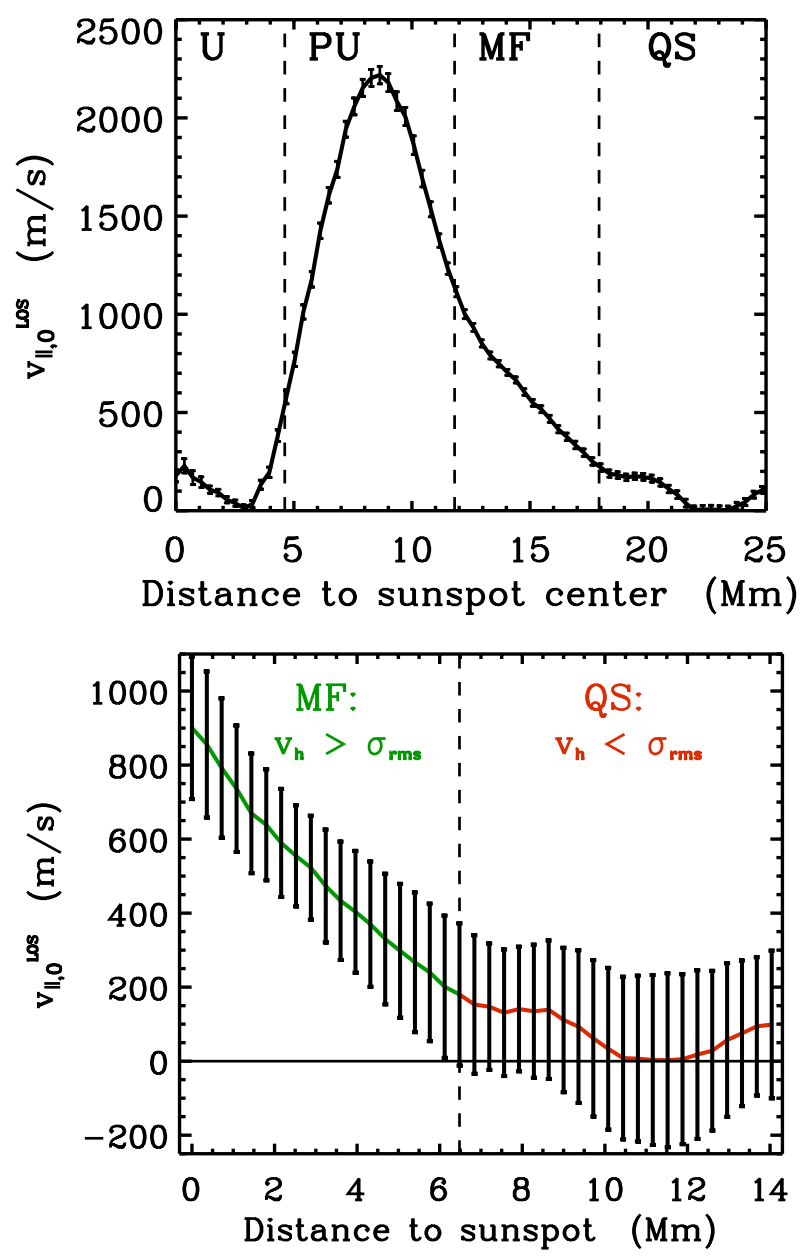

Fig. 8. Upper panel: horizontal flow velocities, $v_{\|, 0}(r)$, in $\mathrm{m} / \mathrm{s}$ for the distance, $r$, from the spot center in Mm. Error bars represent the standard deviation. Dashed vertical lines mark the boundaries between umbra (U), penumbra (PU), and moat flow region (MF) and the quiet Sun (QS). Lower panel: horizontal LOS velocity, $v_{\|, 0}^{\mathrm{LOS}}(r)$, in $\mathrm{m} / \mathrm{s}$ of the MF (green curve) and the quiet Sun (red curve) against the distance from the spot in pixels with $\sigma_{\text {rms }}$ (see Eq. (7)) as error bars.

\subsubsection{Moat flow extension}

To detect the size of the MF region, we define the criteria $v_{\|, 0}^{\mathrm{LOS}} \leq \sigma_{\mathrm{rms}}$ to indicate the end of the detectable MF, whereas the root mean square, $\sigma_{\text {rms }}$, is calculated for each distance, $r$, to the spot by

$\sigma_{\mathrm{rms}}(r)=\sqrt{\frac{1}{n} \cdot \sum_{i=1}^{n}\left(v^{\mathrm{LOS}}\left(r, \phi_{i}\right)-f\left(\phi_{i}\right)\right)^{2}}$.

In the lower panel of Fig. 8, we display $\sigma_{\text {rms }}(r)$ as error bars to the horizontal LOS velocities. When $\sigma_{\text {rms }}(r)$, i.e. the velocity fluctuation around the sine fit, $f\left(\phi_{i}\right)$, with circular angles, $\phi_{i}$ (see Fig. 7), exceeds the fitted amplitude, $v_{\|, 0}^{\text {LOS }}$, we define this distance as the end of the MF region. For the case under consideration, we obtain an MF, which extends $6.5 \mathrm{Mm}$ into the vicinity of the sunspot. This study of the MF extension highly depends on $\sigma_{\text {rms }}$, which is about $180 \mathrm{~m} / \mathrm{s}$ for the $3 \mathrm{~h}$ average and which becomes smaller for longer periods. But, these longer periods cannot guarantee the stability of the observed components. We therefore believe that our method delivers a good estimate for the MF extension. 


\subsection{Evershed flow}

Also the well-known penumbral Evershed flow is a predominantly horizontal flow. In our analysis we assume it as axially symmetrical. In Fig. 8 (upper panel), we display the radial dependence of the horizontal flow component of AR11084 (see Fig. 6c) based on $v_{3 \mathrm{~h}}$. The inner penumbral boundary is determined as the average extension of the umbral darkening in all $720 \mathrm{~s}$ intensity maps. We observe EF velocities, $v_{\|, 0}(r)$, increasing from the inner penumbra to a maximum value of $2210 \mathrm{~m} / \mathrm{s}$ at a distance $r=8.5 \mathrm{Mm} \approx 0.76 \cdot R_{\mathrm{S}}$ followed by a decrease to about $1150 \mathrm{~m} / \mathrm{s}$ at the outer penumbra. Based on our analysis method, some penumbral filaments will reach into the defined MF region or end earlier. Therefore, the velocities at the transition region have impacts on both flows, and therefore the transition is smooth as seen in Fig. 8. But, following the run of the curve, a kink between the radially decreasing EF and MF velocities is evident at the outer penumbral boundary. The mere existence of the kink is a hint at the separation of both flow regions.

\section{Results and discussion}

Overview: in this section, we compare the analysis results (see Sect. 3) for all 51 velocity maps $v_{3 \mathrm{~h}}$ listed in Table A.1. We discuss the interactions between the sunspots and their moat flows. The weekly evolution of the observed components is studied for a sample of 20 sunspots, whereas the monthly evolution is analyzed for three sunspots. We compare our findings with recent studies and analyze the relation of MF properties with sunspot properties. Our discussion is focused on whether the EF and MF are related and on what we can learn about the physical origin of the MF.

\subsection{Horizontal moat flow velocity: $v_{\mathrm{MF}}(r)$}

In the appendix, Figs. A.1 and A.2, we display the radial dependence of the horizontal flow component for all 51 sunspot maps. Since this is the first time that the EF and MF are analyzed coherently for more than only a few spots, it is very remarkable to find that all our spots behave very similarly. The radial dependence is qualitatively the same for all spots. At first glance, only the sunspot size and the maximum of the EF velocity vary. As described in Sect. 3.4 the kink between the velocity decrease of the EF in the penumbra (PU) and the MF at the spot boundary is recognizable for all sunspots in the radial dependences displayed in Figs. A.1 and A.2. In the next step, we examine the flow properties of all spots in detail and compare them.

The moat flow was identified for all selected sunspots as a predominantly horizontal, axially symmetrical flow starting just outside of the penumbra with velocities depending on the distance, $r$, to the sunspot. Figure 9 displays the strong decay of the MF velocities, $v_{\mathrm{MF}}(\tilde{r})=v_{\|, 0}(\tilde{r})$ for all 51 analyzed MF regions based on $v_{3 \mathrm{~h}}$. The upper panel shows the decrease with respect to the distance from the spot center in the radius ratio, $\tilde{r}=\frac{R_{\mathrm{S}}+r}{R_{\mathrm{S}}}$. The lower panel plots the MF velocities against the distance, $\tilde{r}=r=0, \ldots, 14 \mathrm{Mm}$, from the penumbral boundary. The monotone decrease from maximum velocities of $v_{\mathrm{MF}}(r=0)=800 \ldots 1200 \mathrm{~m} / \mathrm{s}$ just outside the sunspot is largely similar for all sunspots. The average run, $\left\langle v_{\mathrm{MF}}(\tilde{r})\right\rangle_{51 \text { spots }}$, starts with $1000 \mathrm{~m} / \mathrm{s}$ just beyond the penumbral boundary at $r=0 \mathrm{Mm}$ and decreases to approx. $500 \mathrm{~m} / \mathrm{s}$ within $r=4 \mathrm{Mm}$ or $\tilde{r}=1.3 \cdot R_{\mathrm{S}}$. The average velocity falls below the typical rms values, $\sigma_{\mathrm{rms}} \approx 180 \mathrm{~m} / \mathrm{s}$, at $r=9.2 \mathrm{Mm}$ or $\tilde{r}=1.7 \cdot R_{\mathrm{S}}$. Consequently, the MF velocity highly depends on the location
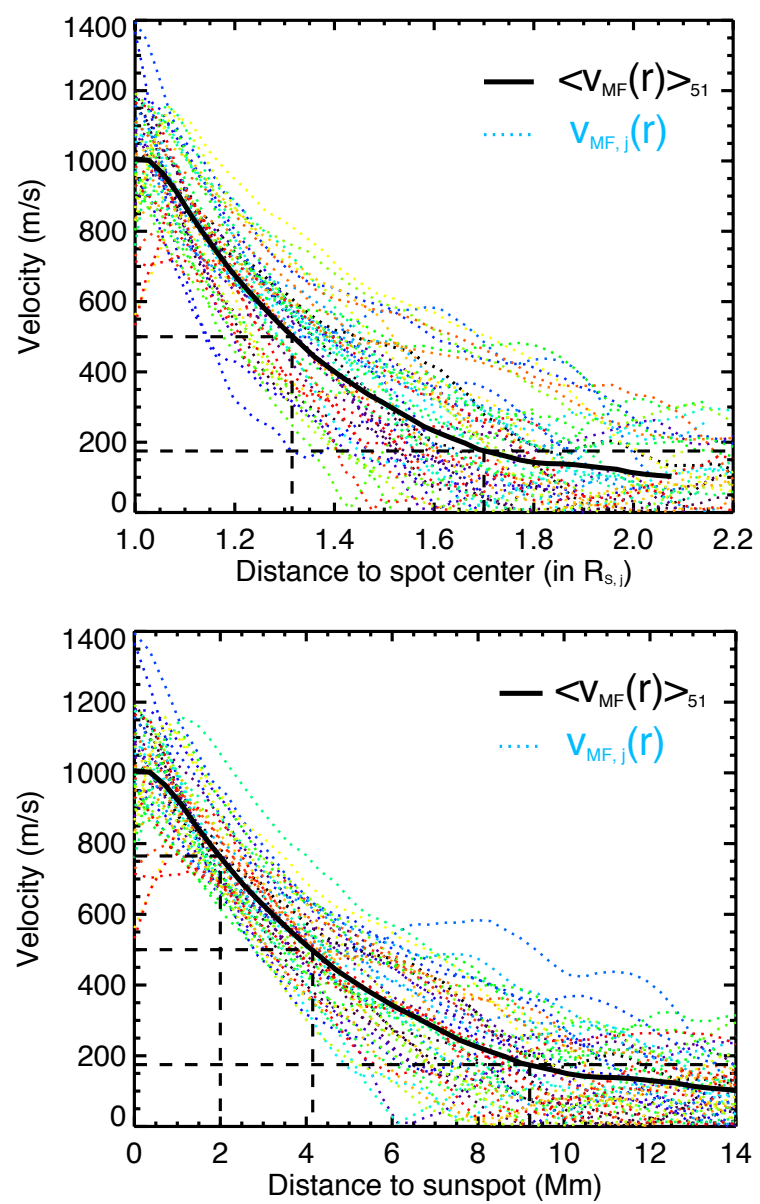

Fig. 9. Horizontal MF velocities, $v_{\mathrm{MF}}(r)$, in $\mathrm{m} / \mathrm{s}$ of 51 observed $3 \mathrm{~h}$ velocity maps against the distance from the spot center in spot ratio (upper panel) and the distance to the sunspot in Mm (lower panel). The calculated average (black bold curve) is drawn.

within the MF region. We compare both panels of Fig. 9 and notice that the runs according to the radius ratio in the left hand panel are spread wider than according to the sheer distance from the spot. Consequently, the MF appears to be independent of the spot size.

This result is in line with recent studies describing the MF velocity on the surface ranging between $v_{\mathrm{MF}}=500 \ldots 1000 \mathrm{~m} / \mathrm{s}$ (Balthasar et al. 1996; Rimmele 1997; Vargas Domínguez et al. 2007), whereas the continuous velocity decrease with increasing distance from the spot boundary has not been measured before.

\subsection{Maximum Evershed flow velocity: $v_{\mathrm{EF}}$}

The maximum EF velocity, $v_{\mathrm{EF}}=\max \left[v_{\|, 0}\left(R_{\mathrm{U}} \leq r \leq R_{\mathrm{S}}\right)\right]$, for all observed penumbrae is in the range of $v_{\mathrm{EF}}=1830 \ldots 3000 \mathrm{~m} / \mathrm{s}$ (Fig. 10e) with an average of $2325 \mathrm{~m} / \mathrm{s}$. Since we measure azimuthally averaged flow speeds of $3 \mathrm{~h}$ averages, our maximum EF velocities are slightly lower than the maximum velocities of up to $3 \ldots 4 \mathrm{~km} \mathrm{~s}^{-1}$ found in recent studies (Schlichenmaier \& Schmidt 2000; Shine et al. 1994; Rouppe van der Voort 2002; Bellot Rubio et al. 2003a). The location, $r_{\mathrm{EF}}$, of the extreme EF velocity can by found at $0.65 R_{\mathrm{S}} \leq r_{\mathrm{EF}} \leq 0.87 R_{\mathrm{S}}$, with an average ratio of $r_{\mathrm{EF}}=0.78 R_{\mathrm{S}}$ which is not as far in the outer spot boundary as $r_{\mathrm{EF}}=0.8 \ldots 0.9 R_{\mathrm{S}}$ (Tritschler et al. 2004; Franz 2011). The velocity run within the penumbra is largely similar for 


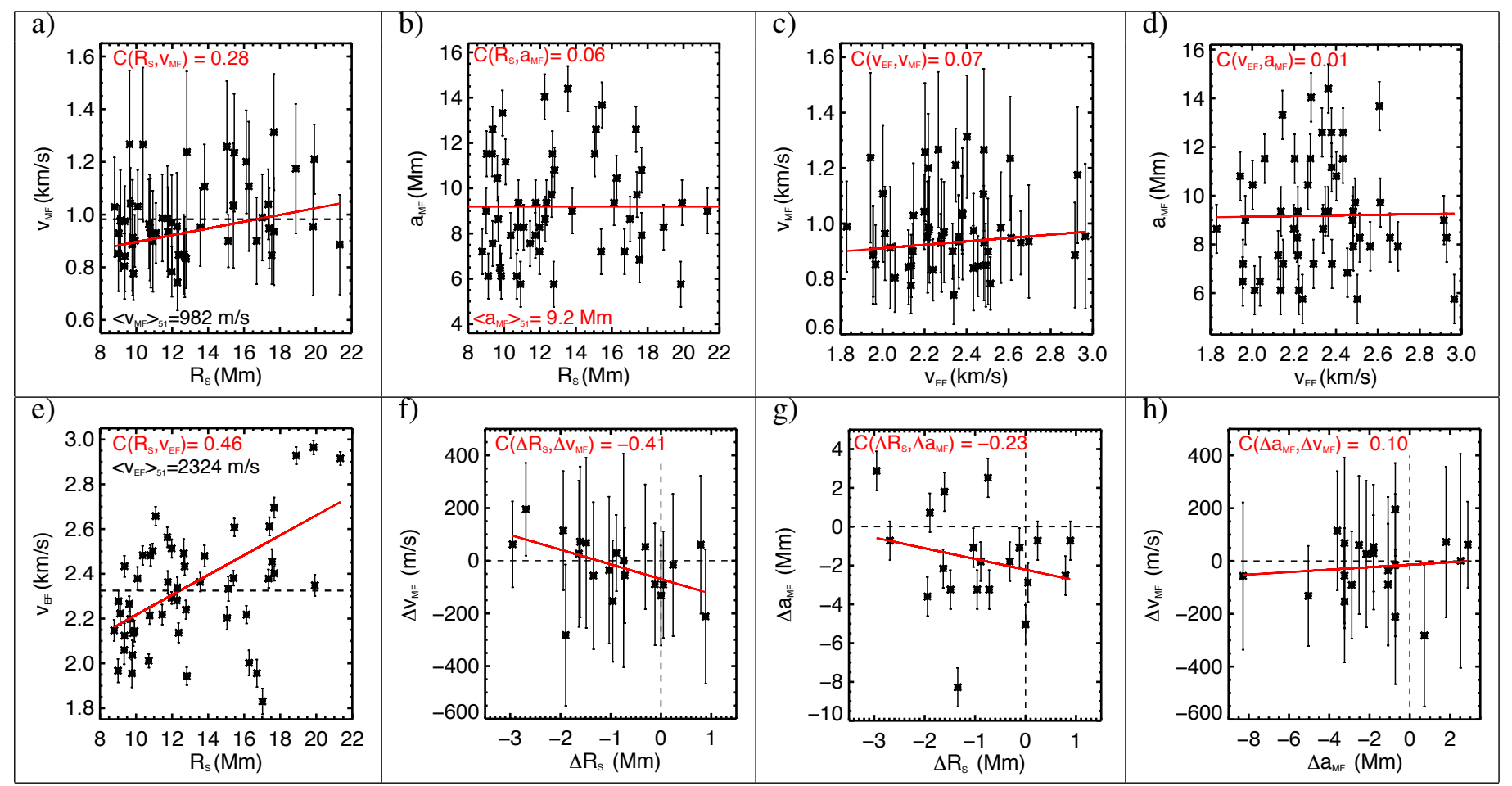

Fig. 10. Correlations of sunspot and MF properties for all 51 sunspot maps (a)-e)), $v_{3 \mathrm{~h}}$, and their evolution within one week for 20 sunspots (f)-h)) indicated by linear regressions (red lines) and the coefficients, $C(\cdot, \cdot)$. a) MF velocities, $v_{\mathrm{MF}}$, in $\mathrm{m} / \mathrm{s}$ and b) MF extensions, $a_{\mathrm{MF}}$, in Mm against the sunspot radii, $R_{\mathrm{S}}$, in Mm with the average values, $\left\langle v_{\mathrm{MF}}\right\rangle_{51}=982 \mathrm{~m} / \mathrm{s}$ and $\left\langle a_{\mathrm{MF}}\right\rangle_{51}=9.2 \mathrm{Mm}$. c) MF velocities, $v_{\mathrm{MF}}$, in m/s and d) MF extensions, $a_{\mathrm{MF}}$, in Mm against the maximum EF velocities, $v_{\mathrm{EF}}$, in $\mathrm{m} / \mathrm{s}$. e) Maximum EF velocities, $v_{\mathrm{EF}}$, in $\mathrm{m} / \mathrm{s}$ against $R_{\mathrm{S}}$ in Mm with the average value, $\left\langle v_{\mathrm{EF}}\right\rangle_{51}=2324 \mathrm{~m} / \mathrm{s}$. f) Weekly changes, $\Delta v_{\mathrm{MF}}$, in $\mathrm{m} / \mathrm{s}$ and $\left.\mathbf{g}\right) \Delta a_{\mathrm{MF}}$ in Mm of the moat flow against $\Delta R_{\mathrm{S}}$ in Mm. h) Weekly changes of MF properties, $\Delta v_{\mathrm{MF}}$, in $\mathrm{m} / \mathrm{s}$ against $\Delta a_{\mathrm{MF}}$ in $\mathrm{Mm}$.

all sunspots with some differences in the skewness as seen in Figs. A.1 and A.2.

\subsection{Correlations}

In the following section, we analyze the correlations between $v_{\mathrm{MF}}, a_{\mathrm{MF}}, v_{\mathrm{EF}}$, and $R_{\mathrm{S}}$ based on the $3 \mathrm{~h}$ average sample.

\subsubsection{Correlation of spot size and MF velocity}

In order to work out the interaction of the sunspots with their moat flows, we plot the MF velocities, $v_{\mathrm{MF}}=\left\langle v_{\mathrm{MF}}(r)\right\rangle_{r=0, \ldots, 5 \mathrm{px}}$, against the sunspot size, $R_{\mathrm{S}}$, as it can be seen in Fig. 10a with a nonsignificant rise of the linear regression (red line) and a correlation coefficient of $C\left(v_{\mathrm{MF}}, R_{\mathrm{S}}\right)=0.28$. Hence, we find that the MF velocity is in average around $1000 \mathrm{~m} / \mathrm{s}$ and does not depend on the spot size. Since we measure close to the outer sunspot boundary the indicated slight increase may be due to a stronger EF in bigger sunspots (see Sects. 4.3.3 and 4.3.4).

\subsubsection{Correlation of spot size and MF extension}

According to Eq. (7) the MF extensions, $a_{\mathrm{MF}}$, were determined for all sunspots with a boundary criteria of $\sigma_{\text {rms }} \approx 180 \mathrm{~m} / \mathrm{s}$ and range between $a_{\mathrm{MF}}=5 \ldots 15 \mathrm{Mm}$ for the spot sizes of $R_{\mathrm{S}}=8.6 \ldots 21.2 \mathrm{Mm}$, cf. Fig. 10b. Since the linear regression and the correlation coefficient of $C\left(a_{\mathrm{MF}}, R_{\mathrm{S}}\right)=0.06$ indicate uncorrelated components, we average the MF extensions to $\left\langle a_{\mathrm{MF}}\right\rangle_{51 \text { spots }}=9.2 \mathrm{Mm}$. Comparing our results to other studies (e.g., Brickhouse \& Labonte 1988) the MF extension lies below the mentioned $a_{\mathrm{MF}}=10 \ldots 20 \mathrm{Mm}$ for small sunspots and far below the double spot radius for larger spots. In our studies, the MF extension is independent of the spot size and therefore is in the order of the sunspot size only for small but not for bigger spots. This finding goes in line with studies by Sobotka \& Roudier (2007). In summary it can be stated that we find four indications that the MF is not correlated to the spot size: (i) the kink of $v_{\|, 0}(r)$ at the outer spot boundary (e.g. Fig. 8: upper panel), (ii) the smaller spread of all 51 MFs by merely displaying them with their radial distance from the spot in Fig. 9, (iii) the trend in Fig. 10a and (iv) the trend in Fig. 10b.

\subsubsection{Correlation of spot size and EF velocity}

To yield the correlation of the sunspot properties, i. e. the maximum horizontal EF velocity between $v_{\mathrm{EF}}=1830 \ldots 3000 \mathrm{~m} / \mathrm{s}$ and the sunspot radius, we plot both components against each other in Fig. 10e and fit a linear regression by considering all values. We obtain a slightly positive trend for bigger sunspots harboring higher maximum EF velocities. Following the regression, sunspots with $R_{\mathrm{S}}=10 \mathrm{Mm}$ would have maximum EF velocities of $2200 \mathrm{~m} / \mathrm{s}$ whereas sunspots with the double size yield maximum EF velocities that are almost $500 \mathrm{~m} / \mathrm{s}$ faster. Although there are some outliers between $16-17 \mathrm{Mm}$, even the correlation coefficient, $C\left(a_{\mathrm{MF}}, R_{\mathrm{S}}\right)=0.46$, suggests a positive link of the EF velocity with the spot size.

\subsubsection{Correlation of Evershed flow and moat flow}

As we pointed out in Sects. 4.3.1 and 4.3.2 the moat flow seems to be independent of the sunspot radius. In the following, we examine the largely unknown link between the penumbral EF and 
Table 1. Monthly evolution for three long-lasting sunspots.

\begin{tabular}{lcccc}
\hline \hline $\begin{array}{l}\text { Spot-No. } \\
\text { (Table A.1) }\end{array}$ & $\begin{array}{c}\Delta R_{\mathrm{S}} \\
(\mathrm{Mm})\end{array}$ & $\begin{array}{c}\Delta a_{\mathrm{MF}} \\
(\mathrm{Mm})\end{array}$ & $\begin{array}{c}\Delta v_{\mathrm{MF}} \\
(\mathrm{m} / \mathrm{s})\end{array}$ & $\begin{array}{c}\Delta v_{\mathrm{EF}} \\
(\mathrm{m} / \mathrm{s})\end{array}$ \\
\hline $4 \rightarrow 6$ & -2.8 & -3.1 & +77 & -65 \\
$7 \rightarrow 9$ & -6.1 & +6.6 & +173 & -455 \\
$11 \rightarrow 13$ & -5.2 & -5.4 & +64 & -115 \\
\hline
\end{tabular}

the adjacent MF. It is therefore important to find out whether higher EF velocities also cause higher MF velocities or a bigger MF extension, which would argue for a partial drive of the MF by the EF. Due to Figs. 10c-d which plot the MF velocities, $v_{\mathrm{MF}}$, and MF extensions, $a_{\mathrm{MF}}$, against the EF velocities, $v_{\mathrm{EF}}$, with correlation coefficients of $C\left(v_{\mathrm{MF}}, v_{\mathrm{EF}}\right)=0.07$ and $C\left(a_{\mathrm{MF}}, v_{\mathrm{EF}}\right)=0.01$, we draw the conclusion that the EF has no impact on the MF. This means that there is a greater decrease in speed for spots with higher maximum EF velocities toward the outer penumbral boundary (see Figs. A.1 and A.2). Although the existence of the MF is directly coupled to the existence of a penumbra and its EF, the observed properties indicate the MF and the EF are independent sunspot flows.

\subsection{Weekly evolution}

To study the temporal evolution of the analyzed components, i.e. $R_{\mathrm{S}}, v_{\mathrm{MF}}, a_{\mathrm{MF}}$, and $v_{\mathrm{EF}}$, we have tracked 20 sunspots (see Table A.1) from the eastern to the western limb sides and performed the analysis of $v_{3 \mathrm{~h}}$ for a second time at $\theta \approx 50^{\circ}$. According to the latitude and the differential rotations, the observing dates range between 6 to 8 days.

The weekly changes are in the range of $\Delta R_{\mathrm{S}}=-3 \ldots 1 \mathrm{Mm}$, $\Delta v_{\mathrm{EF}}= \pm 500 \mathrm{~m} / \mathrm{s}, \Delta v_{\mathrm{MF}}= \pm 200 \mathrm{~m} / \mathrm{s}$ and $\Delta a_{\mathrm{MF}}=-8 \ldots 3 \mathrm{Mm}$. Figures 10f-g plot the changes, $\Delta v_{\mathrm{MF}}$ and $\Delta a_{\mathrm{MF}}$, of the MF velocities and extensions against the change of the spot radius, $\Delta R_{\mathrm{S}}$, with linear regressions according to the correlation coefficients, $C\left(\Delta v_{\mathrm{MF}}, \Delta R_{\mathrm{S}}\right)=-0.41$ and $C\left(\Delta a_{\mathrm{MF}}, \Delta R_{\mathrm{S}}\right)=-0.23$, indicating slightly negative trends. Following this, the MF velocity increases for strongly decaying sunspots and tends to decrease slightly for small $\Delta R_{\mathrm{S}}$. The MF extension, $a_{\mathrm{MF}}$, decreases for the majority of the sunspots with some exceptions for strongly decaying sunspots, but yields no significant correlation with the sunspot evolution. Figure 10h plots the change in the MF velocities, $\Delta v_{\mathrm{MF}}$, against the changing MF extension, $\Delta a_{\mathrm{MF}}$, with no significant correlation, $C\left(\Delta v_{\mathrm{MF}}, \Delta a_{\mathrm{MF}}\right)=0.10$. Because they are $C\left(\Delta v_{\mathrm{EF}}, \Delta R_{\mathrm{S}}\right)=-0.06, C\left(\Delta v_{\mathrm{MF}}, \Delta v_{\mathrm{EF}}\right)=0.23$, $C\left(\Delta a_{\mathrm{MF}}, \Delta v_{\mathrm{EF}}\right)=-0.18$, all other correlations are insignificant and yield no further impact. According to these results, we would draw the conclusions, that a strong sunspot decay leads to an additional drive of the moat flow by accelerating its velocity and sporadically expanding its outreach, whereas it has no impact on the EF velocity.

\subsection{Monthly evolution}

Tracking three long-lasting sunspots across the far side of the Sun, they reappear at the eastern front side (Table A.1: No. $4 \rightarrow 6,7 \rightarrow 9,11 \rightarrow 13$; Figs. 6e-h). This allows us to study the long-term evolution for one month in order to crosscheck the results of the weekly evolution. The changes in sunspot size, $\Delta R_{\mathrm{S}}$, the MF velocity, $\Delta v_{\mathrm{MF}}$, the MF extension, $\Delta a_{\mathrm{MF}}$, and the maximum EF velocity, $\Delta v_{\mathrm{EF}}$, as listed in Table 1 are based on the mean difference between the first and second appearances.
Coupled to the strong decay in sunspot sizes, the MF velocity significantly increases, whereas the EF velocity shows a decrease of several hundred $\mathrm{m} / \mathrm{s}$. These trends are in line with the correlations for the weekly evolution. The size of the MF region shows no unique trend, but the huge widening $\Delta a_{\mathrm{MF}}=+6.6 \mathrm{Mm}$ of Spot-No. 7 (in Table 1) by more than $6 \mathrm{Mm}$ could underline an additional outflow of plasma over the moat cell due to a strong sunspot decay.

\section{Conclusion}

\subsection{Conclusions on the moat flow}

Meyer et al. (1979) suggested that a sunspot is embedded in a supergranular cell. If one puts MF cells in a context with supergranules, one should realize that they have a diameter between 30 and $60 \mathrm{Mm}$, i.e., are up to twice as large as supergranules. Also, the MF velocities are more than twice as high as supergranular velocities (e.g., Brickhouse \& Labonte 1988). As a result, the MF needs a driving mechanism that is distinct from the driving mechanism of normal supergranules. Nye et al. (1988) modeled the MF being driven by a surplus gas pressure beneath the penumbra, arguing that the lack of radiative cooling beneath the penumbra generates a surplus of heat, hence gas pressure. In these models the MF velocity depends on the depth of the penumbra, but not on the size of a spot. At that time this posed a problem, as it was commonly believed that the MF extension scales with the spot radius. Later, Sobotka \& Roudier (2007) provided evidence that there is no correlation between the MF and the spot radius. Our investigation based on Doppler shift measurements rather than on local correlation tracking, independently confirms these later findings. The evidence therefore supports the moat flow model in which the driving forces are due to surplus gas pressure beneath sunspots.

A moat flow that is driven from beneath the penumbra would naturally push away the granules from the spot as seen with local correlation techniques. This implies that the MF is present in the deep photosphere. In contrast, the Evershed flow or the fraction of it that extends from the penumbra outwards into the moat is present in the magnetic canopy that surrounds the sunspot in the mid and upper photosphere (Rezaei et al. 2006). In the immediate surroundings of the sunspot two types of flows exist: (1) the (magnetic) EF that partially continues in the magnetic canopy, which ascends outwards from the mid-photosphere at the spot boundary; (2) the (largely nonmagnetic) MF in the deep photosphere and beneath.

We consider the MMFs to be distinct from the moat flow. They migrate into or are advected by the MF. MMFs are associated with inclined magnetic field lines (relative to horizontal) that reach up into the higher atmospheric layers. These MMF field lines possibly originate in the sunspot and either witness the decay of sunspots (uni-polar MMFs) or are due to some waves that propagate outwards (bipolar MMFs), (see e.g., Sainz Dalda \& Martínez Pillet 2005; Sainz Dalda \& Bellot Rubio 2008a,b; Schlichenmaier 2002). Because of their vertical configuration, outwardly migrating MMFs can also be detected in higher atmospheric layers as reported by Sobotka \& Roudier (2007).

\subsection{Summary}

We calibrated HMI velocity maps such that systematic absolute errors are below $150 \mathrm{~m} / \mathrm{s}$ on an absolute scale. The synoptic CLV of the convective blueshift was measured in good accordance with previous findings and the theoretical synthesis curve. 
We find a maximum velocity of the solar rotation of $1992 \mathrm{~m} / \mathrm{s}$, which agrees with previous measurements and reveals no significant impact by stray light. The instrumental artifacts were identified in line with HMI calibration studies (Centeno et al. 2011). These artifacts make the meridional flow amplitude too small to be measured.

We analyzed $3 \mathrm{~h}$ time averages of sunspot flow velocities. We constructed 51 velocity maps of 31 sunspots. The flow in and around circular sunspots with a fully developed penumbra was analyzed using azimuthal averages, thereby assuming axial symmetry. In both, the MF and EF, the horizontal velocity component was dominant. The vertical flow components of MF and EF were determined to be small. Since the exact amount of convective blueshift is unknown, we do not address the sign of vertical flows.

The radial dependence of the velocity fields was observed to be similar for all 51 sunspot maps. The analysis of the EF yielded results that are consistent with recent studies. A higher EF velocity was detected for bigger sunspots, and the EF velocity scales with the spot size. The MF is a convective outflow with radially decreasing velocity. The MF velocity turned out to be similar in size and independently of the spot size. Also, the MF extension is not correlated with any sunspot property. As MF and EF properties turn out to be uncorrelated, we inferred that the driving mechanisms of the two flows should be distinct. Therefore, we favor the MF model of Nye et al. (1988): the MF is driven by surplus gas pressure beneath the penumbra.

We find a tendency toward increasing MF velocity for strongly decaying sunspots on time scales of one week (spot rotates from the east to west limbs) and one month (spot reappears on the east limb). It is beyond the scope of this paper to put this into the context of a model for decaying sunspots.

Acknowledgements. The HMI instrument of NASA's SDO mission is operated by the HMI/AIA Joint Science Operations Center (JSOC) at Stanford University. The data was provided by the JSOC webpage. We want to thank H.-G. Ludwig for the spectral line synthesis, R. Centeno and S. Couvidat for their correspondence on HMI questions, and J. M. Borrero for his fruitful comments on the manuscript.

\section{References}

Balthasar, H. 1985, Sol. Phys., 99, 31

Balthasar, H., \& Muglach, K. 2010, A\&A, 511, A67

Balthasar, H., Schleicher, H., Bendlin, C., \& Volkmer, R. 1996, A\&A, 315, 603 Beeck, B., Collet, R., Steffen, M., et al. 2012, A\&A, 539, A121

Brickhouse, N. S., \& Labonte, B. J. 1988, Sol. Phys., 115, 43

Bellot Rubio, L. R., Balthasar, H., Collados, M., \& Schlichenmaier, R. 2003a, A\&A, 403, 47

Cabrera Solana, D., Bellot Rubio, L. R., Beck, C., \& del Toro Iniesta, J. C. 2006, ApJ, 649, L41
Cabrera Solana, D., Bellot Rubio, L. R., Beck, C., \& del Toro Iniesta, J. C. 2007, A\&A, 475, 1067

Centeno, R., Tomczyk, S., Borrero, J. M., et al. 2011, Proc. Sol. Polarisation Workshop 6, 437, 147

Cruz Rodríguez, J., de La Kiselman, D., \& Carlsson, M. 2011, A\&A, 528, A113

Delbouille, L., Roland, G., \& Neven, L. 1990, Atlas photometrique DU spectre solaire, Université de Liège

Evershed, J. 1909, MNRAS, 69, 454

Featherstone, N. A., Hindman, B. W., \& Thompson, M. J. 2011, J. Phys.: Conf. Ser., 271, 012002

Franz, M. 2011, Spectropolarimetry of Sunspot Penumbrae, Cuvillier Göttingen Franz, M., \& Schlichenmaier, R. 2009, A\&A, 508, 1453

Freytag, B., Steffen, M., Ludwig, H.-G., et al. 2012, J. Comp. Phys., 231, 919

Gizon, L., Duvall, T. L., Jr., \& Larsen, R. M. 2000, JA\&A, 21, 339

Gizon, L., Schunker, H., Baldner, C. S., et al. 2009, Space Sci. Rev., 144, 249

Harvey, K., \& Harvey, J. 1973, Sol. Phys., 28, 61

Hathaway, D., \& Rightmire, L. 2010, Science, 327, 1350

Howe, R., Jain, K., Hill, F., et al. 2011, J. Phys.: Conf. Ser., 271, 012060

Komm, R., Howe, R., Hill, F., et al. 2011, J. Phys.: Conf. Ser., 271, 012077

Maltby, P. 1964, Astrophys. Norvegica, 8, 205

Meyer, F., Schmidt, H. U., \& Weiss, N. O. 1979, A\&A, 76, 35

Michard, R. 1951, Ann. Astrophys., 14, 101

Nye, A., Bruning, D., \& LaBonte, B. J. 1988, Sol. Phys., 115, 251

Pardon, L., Worden, S. P., \& Schneeberger, T. J. 1979, Sol. Phys., 63, 247

Rezaei, R., Schlichenmaier, R., Beck, C., \& Bellot Rubio, L. R. 2006, A\&A, 454, 975

Rimmele, T. R. 1994, A\&A, 290, 972

Rimmele, T. R. 1997, ApJ, 490, 458

Rouppe van der Voort, L. H. M. 2002, A\&A, 389, 1020

Sainz Dalda, A., \& Bellot Rubio, L. R. 2008a, A\&A, 481, L21

Sainz Dalda, A., \& Bellot Rubio, L. R. 2008b, A\&A, 481, L21

Sainz Dalda, A., \& Martínez Pillet, V. 2005, ApJ, 632, 1176

Schlichenmaier, R. 2002, Astron. Nachr., 323, 303

Schlichenmaier, R., \& Collados, M. 2002, A\&A, 381, 668

Schlichenmaier, R., \& Schmidt, W. 1999, A\&A, 349, L37

Schlichenmaier, R., \& Schmidt, W. 2000, A\&A, 358, 1122

Schlichenmaier, R., Bellot Rubio, L. R., \& Tritschler, A. 2004, A\&A, 415, 731

Schlichenmaier, R., Rezaei, R., Bello González, N., \& Waldmann, T. A. 2010, A\&A, 512, L1

Sheeley, N. R. Jr. 1969, Sol. Phys., 9, 347

Sheeley, N. R. Jr. 1972, Sol. Phys., 25, 98

Shine, R. A., Title, A. M., Tarbell, T. D., et al. 1994, ApJ, 430, 413

Snodgrass, H. B. 1984, Sol. Phys., 94, 13

Sobotka, M., \& Roudier, T. 2007, A\&A, 472, 277

Sobotka, M., Vázquez, M., Bonet, J. A., Hanslmeier, A., \& Hirzberger, J. 1999, ApJ, 511, 436

Solanki, S. K. 2003, A\&ARv, 11, 153

Stix, M. 2002, The Sun (Berlin: Springer)

Sun, M. T., Chou, D. Y., Lin, C. H., \& the TON Team 1997, Sol. Phys., 176, 59

Tritschler, A., Schlichenmaier, R., Bellot Rubio, L. R., et al. 2004, A\&A, 415, 717

Vargas Domínguez, S., Bonet, J. A., Martínez Pillet, V., et al. 2007, ApJ, 660 , L165

Verma, M., Balthasar, H., Deng, N., et al. 2012, A\&A, 538, A109

Vrabec, D. 1971, in Solar Magnetic Fields, ed. R. Howard, IAU Symp., 43, 329

Vrabec, D. 1974, in Chromospheric Fine Structure, ed. R. G. Athay (Dordrecht: Reidel), IAU Symp., 56, 201

Wiehr, E., \& Degenhardt, D. 1992, A\&A, 259, 313

Pages 11 to 13 are available in the electronic edition of the journal at http://www . aanda.org 
J. Löhner-Böttcher and R. Schlichenmaier: Correlations between sunspots and their moat flows

\section{Appendix A: Analyzed sunspots}

Table A.1. List of the 31 numbered, observed sunspots.

\begin{tabular}{|c|c|c|c|c|c|c|c|}
\hline No. & AR & Obs.-No. & Date & Time & Location & $R_{\mathrm{S}}[\mathrm{Mm}]$ & $\theta\left[^{\circ}\right]$ \\
\hline \multirow[t]{2}{*}{1} & 11084 & $\mathrm{a}$ & $2010-06-29$ & $00: 00$ & S19 E45 & 11.5 & 49 \\
\hline & & $\mathrm{b}$ & 2010-07-06 & $00: 00$ & S19 W47 & 11.2 & 51 \\
\hline 2 & 11092 & $\mathrm{a}$ & 2010-08-06 & $20: 00$ & N13 W44 & 15.1 & 45 \\
\hline \multirow[t]{2}{*}{3} & 11101 & $\mathrm{a}$ & $2010-08-27$ & 00:00 & N13 E45 & 11.5 & 46 \\
\hline & & $\mathrm{b}$ & 2010-09-03 & 08:00 & N12 W48 & 11.9 & 49 \\
\hline \multirow[t]{2}{*}{$4(\rightarrow 6)$} & 11108 & $\mathrm{a}$ & $2010-09-25$ & 00:00 & $\mathrm{S} 30 \mathrm{~W} 36$ & 17.3 & 49 \\
\hline & & $\mathrm{b}$ & $2010-09-25$ & $10: 00$ & $\mathrm{~S} 30 \mathrm{~W} 40$ & 17.3 & 52 \\
\hline \multirow[t]{2}{*}{5} & 11113 & $\mathrm{a}$ & $2010-10-16$ & 00:00 & N17 E48 & 12.6 & 49 \\
\hline & & $\mathrm{b}$ & $2010-10-23$ & $10: 00$ & N16 W48 & 10.4 & 48 \\
\hline \multirow[t]{2}{*}{$6(\leftarrow 4)$} & 11115 & $\mathrm{a}$ & $2010-10-17$ & $18: 00$ & $\mathrm{~S} 28 \mathrm{E} 40$ & 15.1 & 52 \\
\hline & & $\mathrm{b}$ & $2010-10-24$ & 04:00 & S29 W40 & 13.7 & 52 \\
\hline \multirow[t]{2}{*}{$7(\rightarrow 9)$} & 11131 & $\mathrm{a}$ & 2010-12-05 & 00:00 & N30 E39 & 21.2 & 49 \\
\hline & & $\mathrm{b}$ & $2010-12-11$ & 08:00 & N30 W38 & 19.8 & 47 \\
\hline 8 & 11133 & $\mathrm{a}$ & $2010-12-14$ & 04:00 & N14 W49 & 10.1 & 51 \\
\hline \multirow{2}{*}{$9(\leftarrow 7)$} & 11140 & $\mathrm{a}$ & 2011-01-02 & $20: 00$ & N32 E40 & 15.1 & 51 \\
\hline & & $\mathrm{b}$ & 2011-01-08 & $20: 00$ & N33 W35 & 13.3 & 47 \\
\hline \multirow[t]{2}{*}{10} & 11147 & $\mathrm{a}$ & 2011-01-18 & $16: 00$ & $\mathrm{~N} 25 \mathrm{E} 41$ & 12.6 & 45 \\
\hline & & $\mathrm{b}$ & 2011-01-24 & $12: 00$ & $\mathrm{~N} 24 \mathrm{~W} 43$ & 11.9 & 49 \\
\hline $11(\rightarrow 13)$ & 11195 & $\mathrm{a}$ & 2011-04-28 & $10: 00$ & S17 W44 & 15.1 & 48 \\
\hline \multirow[t]{2}{*}{12} & 11203 & $\mathrm{a}$ & 2011-05-01 & $12: 00$ & N18 E45 & 12.2 & 47 \\
\hline & & $\mathrm{b}$ & 2011-05-08 & 09:00 & N17 W47 & 10.8 & 50 \\
\hline \multirow[t]{2}{*}{$13(\leftarrow 11)$} & 11216 & $\mathrm{a}$ & 2011-05-18 & $12: 00$ & S15 E47 & 10.4 & 48 \\
\hline & & $\mathrm{b}$ & $2011-05-25$ & $14: 00$ & S15 W46 & 9.0 & 47 \\
\hline \multirow[t]{2}{*}{14} & 11251 & $\mathrm{a}$ & 2011-07-13 & $15: 00$ & N16 E49 & 12.2 & 49 \\
\hline & & $\mathrm{b}$ & $2011-07-20$ & $20: 00$ & N17 W46 & 9.4 & 46 \\
\hline 15 & 11260 & $\mathrm{a}$ & 2011-08-02 & $18: 00$ & N19 W46 & 17.6 & 50 \\
\hline \multirow[t]{2}{*}{16} & 11277 & $\mathrm{a}$ & 2011-08-27 & 00:00 & N17 E47 & 9.0 & 51 \\
\hline & & $\mathrm{b}$ & 2011-09-03 & 09:00 & N19 W46 & 9.7 & 50 \\
\hline \multirow[t]{2}{*}{17} & 11287 & $\mathrm{a}$ & 2011-09-06 & 00:00 & S31 E37 & 12.2 & 49 \\
\hline & & $\mathrm{b}$ & 2011-09-11 & 18:00 & S29 W39 & 9.0 & 52 \\
\hline 18 & 11305 & $\mathrm{a}$ & 2011-10-04 & $20: 00$ & N11 W48 & 14.8 & 50 \\
\hline \multirow[t]{2}{*}{19} & 11312 & $\mathrm{a}$ & 2011-10-07 & 00:00 & N22 E48 & 17.6 & 49 \\
\hline & & $\mathrm{b}$ & 2011-10-14 & 03:00 & $\mathrm{N} 23 \mathrm{~W} 43$ & 17.3 & 45 \\
\hline 20 & 11314 & $\mathrm{a}$ & 2011-10-19 & 00:00 & N26 W42 & 16.6 & 46 \\
\hline \multirow[t]{2}{*}{21} & 11317 & $\mathrm{a}$ & 2011-10-14 & 00:00 & S27 E42 & 9.4 & 51 \\
\hline & & $\mathrm{b}$ & $2011-10-20$ & 06:00 & S27 W41 & 9.4 & 51 \\
\hline 22 & 11338 & $\mathrm{a}$ & $2011-11-10$ & $17: 00$ & S12 W48 & 12.6 & 53 \\
\hline \multirow[t]{2}{*}{23} & 11340 & $\mathrm{a}$ & 2011-11-07 & 00:00 & S09 E49 & 10.1 & 50 \\
\hline & & $\mathrm{b}$ & 2011-11-14 & $10: 00$ & S08 W49 & 9.4 & 50 \\
\hline 24 & 11342 & $\mathrm{a}$ & 2011-11-15 & 00:00 & N17 W47 & 12.6 & 48 \\
\hline 25 & 11343 & $\mathrm{a}$ & 2011-11-16 & $00: 12$ & N28 W39 & 8.6 & 44 \\
\hline 26 & 11355 & $\mathrm{a}$ & $2011-11-27$ & $21: 00$ & N14 W45 & 9.7 & 47 \\
\hline 27 & 11356 & $\mathrm{a}$ & 2011-11-27 & $21: 00$ & N16 W23 & 10.8 & 30 \\
\hline \multirow[t]{2}{*}{28} & 11366 & $\mathrm{a}$ & 2011-12-05 & 00:00 & N17 E51 & 9.7 & 53 \\
\hline & & $\mathrm{a}$ & 2011-12-12 & 08:00 & N18 W47 & 9.0 & 48 \\
\hline \multirow[t]{2}{*}{29} & 11384 & $\mathrm{a}$ & $2011-12-23$ & 00:00 & N12 E36 & 19.8 & 37 \\
\hline & & $\mathrm{b}$ & $2011-12-26$ & $00: 00$ & N13 W01 & 18.7 & 14 \\
\hline \multirow[t]{2}{*}{30} & 11388 & $\mathrm{a}$ & 2011-12-30 & 00:00 & S23 E42 & 10.8 & 45 \\
\hline & & $\mathrm{b}$ & 2012-01-05 & 00:00 & S24 W35 & 9.7 & 40 \\
\hline 31 & 11389 & $\mathrm{a}$ & $2011-12-31$ & 00:00 & S23 E43 & 15.8 & 45 \\
\hline & & $\mathrm{b}$ & 2012-01-07 & 00:00 & $\mathrm{S} 21 \mathrm{~W} 45$ & 16.9 & 50 \\
\hline
\end{tabular}

Notes. The corresponding NOAA number of the active region (AR) is given. The number, date, and starting time of $3 \mathrm{~h}$ observation, as well as the average spot location, the spot radius, $R_{\mathrm{S}}$, (in $\mathrm{Mm}$ ), and the heliocentric angle, $\theta$ (in ${ }^{\circ}$ ), are listed. The spots with No. $4+6,7+9$, and $11+13$ describe the same recurrent sunspots, respectively. 


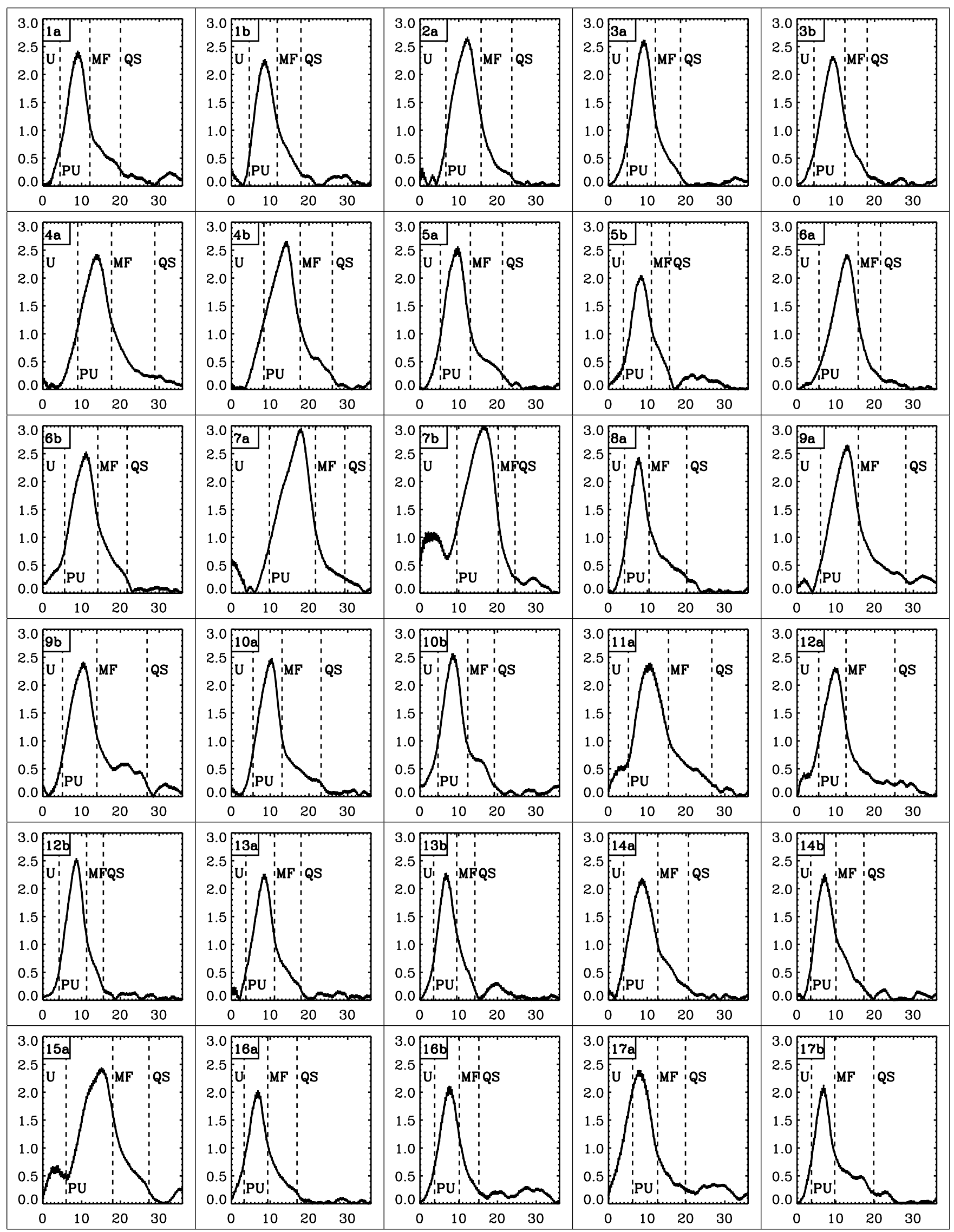

Fig. A.1. Horizontal flow velocities, $v_{\|, 0}(r)$, in $\mathrm{km} \mathrm{s}^{-1}$ from the sunspot center $(r=0 \mathrm{Mm})$ to a distance of $36 \mathrm{Mm}$ in the quiet Sun(QS) for spots No. 1-17 (see Table A.1). The boundaries of the umbra (U), the penumbra (PU), and the end of the moat flow region (MF) are indicated as vertical dashed lines. 
J. Löhner-Böttcher and R. Schlichenmaier: Correlations between sunspots and their moat flows

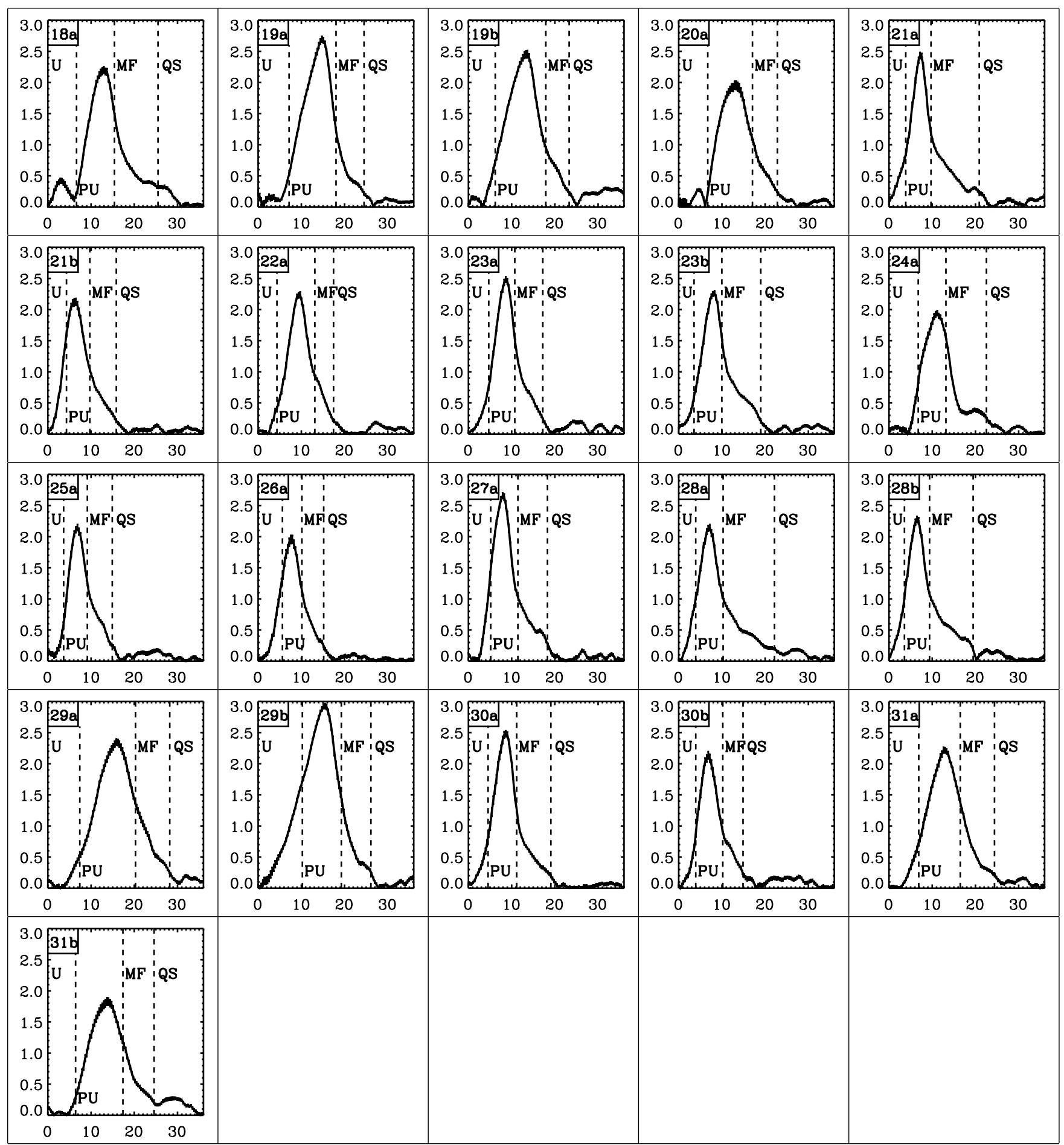

Fig. A.2. Horizontal flow velocities, $v_{\|, 0}(r)$, in $\mathrm{km} \mathrm{s}^{-1}$ from the sunspot center $(r=0 \mathrm{Mm})$ to a distance of $36 \mathrm{Mm}$ in the quiet Sun(QS) for spots No. 18-31 (see Table A.1). The boundaries of the umbra (U), the penumbra (PU), and the end of the moat flow region (MF) are indicated as vertical dashed lines. 